\title{
On classification of intrinsic localized modes for the Discrete Nonlinear Schrödinger Equation
}

\author{
G. L. Alfimov ${ }^{a}, 1$, V. A. Brazhnyi ${ }^{b, *}$, V. V. Konotop ${ }^{c}$ \\ ${ }^{a}$ Departamento de Matemática Aplicada, Facultad de Informatica, Universidad \\ Complutense, 28040, Madrid, Spain \\ ${ }^{\mathrm{b}}$ Centro de Física Teórica e Computacional, Universidade de Lisboa, Av.Prof. \\ Gama Pinto 2, 1649-003 Lisboa, Portugal \\ ${ }^{\mathrm{c}}$ Centro de Física Teórica e Computacional and Departamento de Física, \\ Universidade de Lisboa, Av.Prof. Gama Pinto 2, 1649-003 Lisboa, Portugal
}

\begin{abstract}
We consider localized modes (discrete breathers) of the discrete nonlinear Schrödinger equation $i \frac{d \psi_{n}}{d t}=\psi_{n+1}+\psi_{n-1}-2 \psi_{n}+\sigma\left|\psi_{n}\right|^{2} \psi_{n}, \sigma= \pm 1, n \in \mathbb{Z}$. We study the diversity of the steady-state solutions of the form $\psi_{n}(t)=e^{i \omega t} v_{n}$ and the intervals of the frequency, $\omega$, of their existence. The base for the analysis is provided by the anticontinuous limit ( $\omega$ negative and large enough) where all the solutions can be coded by the sequences of three symbols "-", "0" and "+". Using dynamical systems approach we show that this coding is valid for $\omega<\omega^{*} \approx-3.4533$ and the point $\omega^{*}$ is a point of accumulation of saddle-node bifurcations. Also we study other bifurcations of intrinsic localized modes which take place for $\omega>\omega^{*}$ and give the complete table of them for the solutions with codes consisting of less than four symbols.
\end{abstract}

Key words: Discrete Nonlinear Schrödinger equation, intrinsic localized modes, discrete breather, bifurcations

PACS: 02.30.Oz, 03.75.Lm, 05.45.-a, 63.20.Pw

* Corresponding author

Email addresses: alfimov@nonlin.msk.ru (G. L. Alfimov ), brazhnyi@cii.fc.ul.pt (V. A. Brazhnyi), konotop@cii.fc.ul.pt (V. V. Konotop).

1 On sabbatical leave from Lukin Institute of Physical Problems, 103460, Zelenograd, Moscow, Russia

Preprint submitted to Elsevier Science 31 October 2018 


\section{Introduction}

The discrete nonlinear Schrödinger equation (DNLS)

$$
i \frac{d \psi_{n}}{d t}=\Delta_{2} \psi_{n}+\sigma\left|\psi_{n}\right|^{2} \psi_{n}
$$

where $\psi_{n} \equiv \psi_{n}(t), \Delta_{2} \psi_{n} \equiv \psi_{n+1}-2 \psi_{n}+\psi_{n-1}$, and $\sigma= \pm 1$, is one of the basic lattice models which appeared in last decades in various contexts of physics and biology and has been intensively studied (for review see e.g. $[1,2,3,4]$ ). One of the recent applications of (1) is related to the theory of Bose-Einstein condensates in optical lattices, where the mean-field Gross-Pitaevskii equation with a periodic potential is reduced to (2) within the framework of the so-called tight-binding approximation (see e.g. [5] and references therein). A particular attention has been paid to specific solutions of (1) which are spatially localized and periodic in time and called intrinsic localized modes or, alternatively, discrete breathers. For numerous applications of model (1) it is desirable to have at hands a practical guide where possible forms of intrinsic localized modes were described together with the information about their stability. The aim of this paper is to make a step toward the compilation of this guide.

The simplest class of the discrete breathers can be obtained using the steadystate ansatz $\psi_{n}(t)=e^{i \omega t} u_{n}$. Then the stationary amplitudes $u_{n}, n=0, \pm 1, \pm 2 \ldots$ satisfy the lattice equation

$$
\Delta_{2} u_{n}+\omega u_{n}+\sigma\left|u_{n}\right|^{2} u_{n}=0
$$

and the condition of spatial localization

$$
u_{n} \rightarrow 0 \quad \text { as } \quad n \rightarrow \pm \infty
$$

Eq.(2) is quite general and appears independently in other applications, for example, in polaron theory as the equation for stationary localized solutions (i.e. polarons) derived on the basis of the semiclassical Holstein model [6]. Generically, $\sigma$ can be both positive and negative, $\omega$ can be any real value and the amplitudes $u_{n}, n \in \mathbb{Z}$ can be complex. However, for localized solutions (3) the analysis can be simplified by taking into account the following points:

(a) One can show [1] that all the solutions of Eq.(2) which satisfy the localization condition (3) have the form $u_{n}=v_{n} e^{i \theta}$, where $n \in \mathbb{Z}, v_{n}$ and $\theta$ are real.

(b) The localization condition (3) implies that $\omega$ does not lie in the phonon band defined by $0<\omega<4$. Solutions for $\omega<0$ and for $\omega>4$ are connected 
by the following staggering transform: if discrete function $\left\{u_{n}\right\}, n \in \mathbb{Z}$ is a solution of (2) for $\sigma=\sigma_{0}, \omega=\omega_{0}<0$ then $\left\{(-1)^{n} u_{n}\right\}$ is a solution of (2) for $\sigma=-\sigma_{0}$ and $\omega=4-\omega_{0}$.

(c) One can show [7] that for $\omega<0$ and $\sigma=-1$ (or, correspondingly, for $\omega>4$ and $\sigma=1$ ) all the solutions of (2) except the trivial zero solution are unbounded i.e. $\left|u_{n}\right| \rightarrow \infty$ as $n$ tends either to $+\infty$ or to $-\infty$.

Thus, for the study of localized solutions Eq.(2) can be replaced by

$$
\Delta_{2} u_{n}+\omega u_{n}+u_{n}^{3}=0
$$

where the discrete function $\left\{u_{n}\right\}, n \in \mathbb{Z}$ is real and $\omega<0$.

Solutions of (4) have been widely discussed in physical and mathematical literature. Apart from finding important particular examples, such as the SieversTakeno mode [8], the Page mode [9], the twisted modes [10,11], some authors have addressed the problem of the existence and stability of more complex objects [10]. Recently, the analysis of general structure of the set of localized solutions of Eq.(4) was proposed in [12,13]. In particular, Ref. [12] is devoted to classification of symmetric localized solutions of (4) while in Ref. [13] a quite general approach for the study of both symmetric and non-symmetric solutions of Eq.(4) was elaborated. Both these papers offer a coding for the solutions of Eq.(4). Some discussion of these results in comparison with our ones can be found below in Sect.5.

In the present paper we study different types of localized modes which can coexist at a given parameter $\omega$. The general scheme of our approach is as follows. For large values of $|\omega|$ we describe solutions in terms of coding sequences using anticontinuous limit approach [14]. To this end we rewrite Eq.(4) in the form

$$
\alpha \Delta_{2} v_{n}-v_{n}+v_{n}^{3}=0, \quad \alpha \equiv-1 / \omega
$$

where $u_{n}=\sqrt{-\omega} v_{n}, n \in \mathbb{Z}$. Next, we calculate the boundary $\omega^{*}$ until which this coding works. Finally, we study bifurcations of the solutions, describing them in terms of their coding sequences. This step is implemented using the representation of Eq.(4) as a map in $\mathbb{R}^{2}$. In this part our work was influenced by the paper [15] where a similar problem was analyzed for the Hénon map. The information about the bifurcations of the simplest solutions (the solutions which have codes of length smaller then 4) is collected in Table 1.

The paper is organized as follows. In Section 2 we describe the coding of the solutions which comes from anticontinuous limit and give an estimation for the interval where it is valid. In Section 3 we study the problem from the viewpoint 
of dynamical systems introducing a 2D map $T$ associated with the discrete equation (5). In these terms the problem is reduced to the analysis of the intersection points of stable $W_{s}$ and unstable $W_{u}$ manifolds of zero hyperbolic fixed point of $T$. We establish the ordering of points of intersection on these manifolds. Using this information in Section 4 we describe the homoclinic bifurcations. Section 5 contains a summary and discussion of the results.

\section{Anticontinuous limit}

The anticontinuous limit (ACL) corresponds to the zero value of the coupling constant $\alpha$ in Eq.(5). When $\alpha=0$ the amplitudes of the sites $v_{n}$ are independent from each other and can acquire one of the three values as follows: 0 and \pm 1 . Thus any solution $\left\{v_{n}\right\}$ can be coded by an infinite sequence $\left\{s_{n}\right\}$, $n \in \mathbb{Z}$, where $s_{n}$ is one of the symbols,,- 0 , and + , corresponding to $v_{n}=-1$, $v_{n}=0$ and $v_{n}=1$. Localized solutions in this case can be identified with those of the sequences which start and end with infinite number of consecutive zeros and contain a finite number of nonzero elements (an example is $\{\ldots, 0,0,+,-,+, 0,0, \ldots\})$. We denote the set of all these sequences by $\mathcal{L}_{0}$. It is convenient to define the code and the length of the sequences from $\mathcal{L}_{0}$.

Definition 2.1 A code of a sequence $\{\eta\} \in \mathcal{L}_{0}$ is the maximal subsequence of $\{\eta\}$ which has nonzero first and last elements.

Definition 2.2 A length of a sequence $\{\eta\} \in \mathcal{L}_{0}$ is a number of symbols in its code.

Definition 2.3 A word is a sequence of the symbols $\{-, 0,+\}$. A word which consists of a finite number of symbols is called a finite word.

The above mentioned example $\{\ldots, 0,0,+,-,+, 0,0, \ldots\}$ has the code $\{+-$ $+\}$ and its length is equal to 3 .

It is known [14] that all solutions which exist in the limit $\alpha=0$ can be continued by $\alpha$ up to some positive value $\alpha^{*}$. Moreover, the solutions corresponding to the sequences from $\mathcal{L}_{0}$ remain localized under this continuation and their decay as $n \rightarrow \pm \infty$ is exponential. A basis for the above statements due to [14] is given in Appendix A.

In literature there are various bounds for the limit value $\alpha^{*}$. Application of Theorem 9 of the paper [16] to Eq.(5) yields $\alpha^{*}>1 /(10+4 \sqrt{2}) \approx 0.0639$. The theorem from Section 3.3 of [1] states that $\alpha^{*}>1 / 6 \sqrt{5} \approx 0.0745$. Below we show that $\alpha^{*}>(3 \sqrt{3}-1) / 52 \approx 0.0807$ by making use of the following statement 
Lemma 2.1 For each bounded solution $\mathbf{v}=\left\{v_{n}\right\}, n \in \mathbb{Z}$, of Eq.(5) the following estimation is valid:

$$
\sup _{n \in \mathbb{Z}}\left|v_{n}-v_{n}^{3}\right| \leq 4 \alpha \sqrt{1+4 \alpha}
$$

Proof. Suppose that $\mathbf{v}$ is a solution of (5), (3) and there exists $\rho_{0}>4 \alpha \sqrt{1+4 \alpha}$ and $n_{0}$ such that $\left|v_{n_{0}}-v_{n_{0}}^{3}\right| \geq \rho_{0}$. This implies that $\left|v_{n_{0}+1}-2 v_{n_{0}}+v_{n_{0}-1}\right|>\rho_{0} / \alpha$ and, consequently, there exists $m \in\left\{n_{0}-1, n_{0}, n_{0}+1\right\}$ such that $\left|v_{m}\right|>\rho_{0} / 4 \alpha$ and

$$
\left|v_{m}-v_{m}^{3}\right|>\left(\frac{\rho_{0}}{4 \alpha}\right)^{3}-\left(\frac{\rho_{0}}{4 \alpha}\right) \equiv \rho_{1}>4 \alpha
$$

Repeating the same procedure one can construct the sequence $\rho_{0}, \rho_{1}, \rho_{2}, \ldots$, such that

$$
\rho_{k+1}=\left(\frac{\rho_{k}}{4 \alpha}\right)^{3}-\left(\frac{\rho_{k}}{4 \alpha}\right)
$$

By simple graphical arguments one can show that if $\rho_{0}>4 \alpha \sqrt{1+4 \alpha}$ then $\rho_{k} \rightarrow \infty$ monotonically as $k \rightarrow \infty$. Since $\sup _{n}\left|v_{n}-v_{n}^{3}\right|>\rho_{k}$ for any $k=$ $0,1,2 \ldots$ we arrive to the contradiction which proves the relation (6)

Let $\alpha$ be fixed and $\mathbf{v}=\left\{v_{n}\right\}, n \in \mathbb{Z}$ be a bounded solution of (5). Define the function

$$
M(\alpha) \equiv \sup _{\mathbf{v}} \sup _{n \in \mathbb{Z}} \min \left\{\left|v_{n}\right|,\left|v_{n}-1\right|,\left|v_{n}+1\right|\right\}
$$

where the first supremum is taken over all bounded solutions of (5). Lemma 2.1 implies

Corollary 2.1 The function $M(\alpha)$ is correctly defined and $M(\alpha) \rightarrow 0$ when $\alpha \rightarrow 0$.

Using Lemma 2.1 one can prove also the following statement:

Theorem 2.1 For some interval $0<\alpha<\alpha^{*}$, where $\alpha^{*} \geq \alpha_{0}=(3 \sqrt{3}-1) / 52$ there exists one-to-one correspondence between the set of all localized solutions of Eq.(5) and the set of all sequences from $\mathcal{L}_{0}$.

The proof can be found in Appendix B. Reformulating this statement for initial equation (4) results in the following theorem 

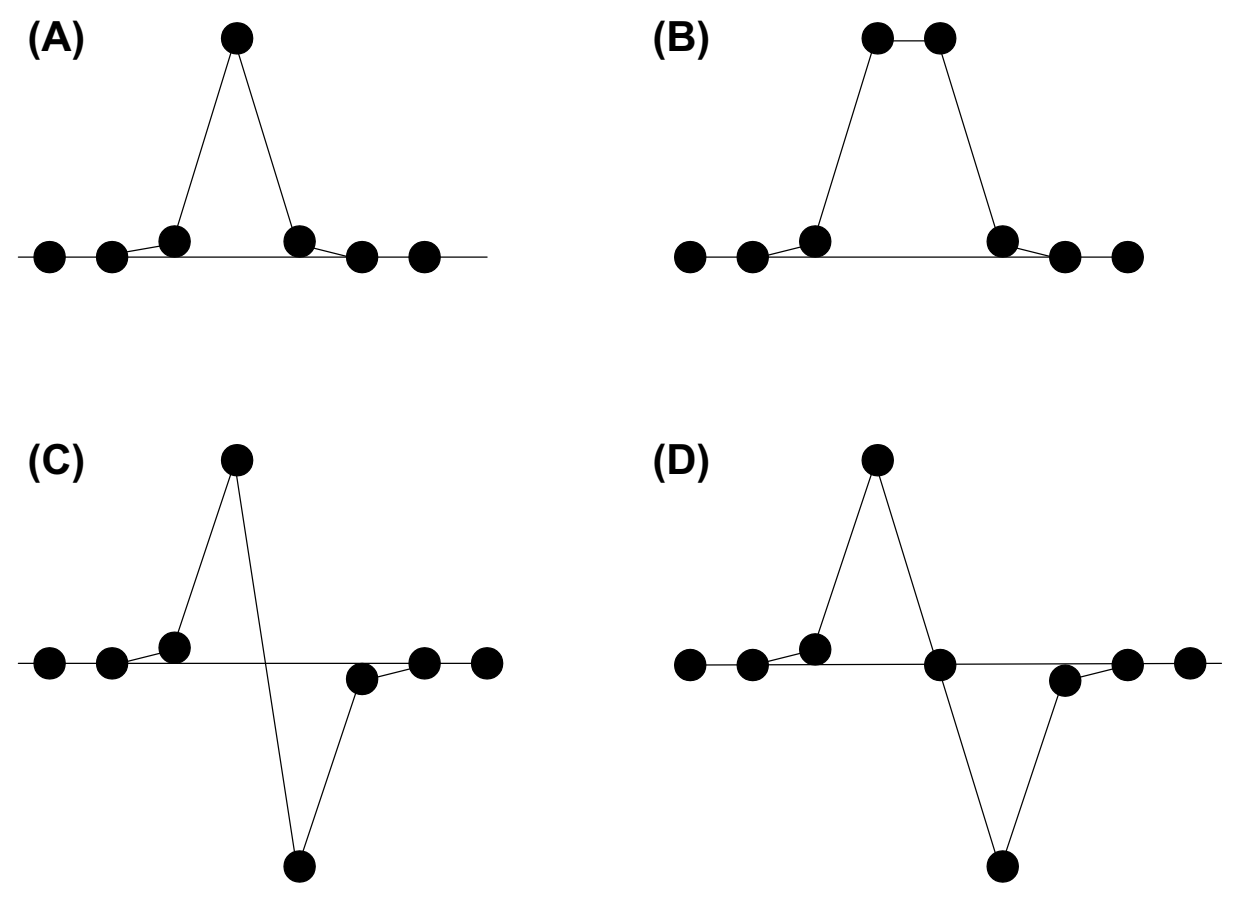

Fig. 1. Some simplest modes of Eq.(5): (a) the Sievers-Takeno mode (the code $\{+\}$ ); (b) the Page mode (the code $\{++\}$ ); (c,d) the twisted modes (the codes $\{+-\}$ and $\{+0-\})$.

Theorem 2.2 For some interval $-\infty<\omega<\omega^{*}$, where $\omega^{*} \geq \omega_{0}=-52 /(3 \sqrt{3}-$ $1) \approx-12.3923$ there exists one-to-one correspondence between the set of all localized solutions of Eq.(4) and the set of all sequences from $\mathcal{L}_{0}$. As $\omega \rightarrow-\infty$ the amplitudes of the sites denoted by $\{ \pm\}$ tend to $\pm \sqrt{-\omega}$, whereas the amplitudes of the sites denoted by $\{0\}$ tend to 0 .

Theorem 2.1 establishes a correspondence between the localized solutions of (5) and the codes of the sequences from $\mathcal{L}_{0}$ for $\alpha$ "close " to anticontinuous limit. We call this correspondence $A C L$ coding. As examples of such coding we mention (see Fig.1) that the Sievers-Takeno mode has the code $\{+\}$ (or $\{-\}$ ), the Page mode can be coded by $\{++\}$ (or $\{--\}$ ) and the twisted modes of two various types have the codes $\{+-\}$ (or $\{-+\})$ and $\{+0-\}($ or $\{-0+\})$, correspondingly.

The estimation for $\alpha^{*}$ given above is not optimal. In the Section 4 we calculate $\alpha^{*}$ numerically. For this we use the dynamical system approach. 


\section{The dynamical system approach}

\subsection{Basic notations and some auxiliary statements}

It is convenient to present the real-valued second-order difference equation (5) in a form of a two-dimensional area-preserving map $T[1,3,17,18]$

$$
T:\left\{\begin{array}{l}
\tilde{x}=y \\
\tilde{y}=\left(2+\frac{1}{\alpha}-\frac{1}{\alpha} y^{2}\right) y-x .
\end{array}\right.
$$

The inverse map $T^{-1}$ is given by

$$
T^{-1}:\left\{\begin{array}{l}
x=\left(2+\frac{1}{\alpha}-\frac{1}{\alpha} \tilde{x}^{2}\right) \tilde{x}-\tilde{y} \\
y=\tilde{x}
\end{array}\right.
$$

In what follows we call a point $\mathbf{b}=T \mathbf{a} \in \mathbb{R}^{2}$ the $T$-image of the point $\mathbf{a} \in \mathbb{R}^{2}$. The $T$-image of a curve in $\mathbb{R}^{2}$ is defined as a set consisting of $T$-images of all points of the curve.

The map $T$ is associated with Eq.(5). Specifically, if $\mathbf{a}=\left(x_{0}, y_{0}\right) \in \mathbb{R}^{2}$ is an arbitrary point of the plane, then the bi-infinite vector formed by $x$-coordinates of points $T^{n} \mathbf{a}, n \in \mathbb{Z}$ satisfies Eq.(5). The map $T$ admits the following inversions

$$
\begin{aligned}
T^{-1}\left(\begin{array}{l}
x \\
y
\end{array}\right) & =\left(\begin{array}{ll}
0 & 1 \\
1 & 0
\end{array}\right) T\left(\begin{array}{l}
y \\
x
\end{array}\right), \\
T\left(\begin{array}{r}
-x \\
-y
\end{array}\right) & =\left(\begin{array}{rr}
-1 & 0 \\
0 & -1
\end{array}\right) T\left(\begin{array}{l}
x \\
y
\end{array}\right) .
\end{aligned}
$$

For $\alpha>0$ and $\alpha<-1 / 4$ the point $\mathcal{O}=(0,0)$ is a hyperbolic fixed point (see e.g. [1]) of $T$. It possesses invariant stable, $W_{s}$, and unstable, $W_{u}$, manifolds. Both $W_{u}$ and $W_{s}$ consist of two branches (see Fig.2): $W_{s}=W_{s}^{+} \cup W_{s}^{-}$and $W_{u}=W_{u}^{+} \cup W_{u}^{-}$. We denote by $W_{u}^{+}$the branch of manifold $W_{u}$ which goes out from the hyperbolic fixed point $\mathcal{O}$ keeping $y$ positive. Correspondingly, $W_{s}^{+}$is the branch of manifold $W_{s}$ which enters the hyperbolic fixed point $\mathcal{O}$ with positive $x$. The branches are connected by the symmetries: $W_{u, s}^{+}$and $W_{u, s}^{-}$ are symmetric with respect to $\mathcal{O}$ whereas $W_{s, u}^{ \pm}$and $W_{u, s}^{ \pm}$are symmetric with 


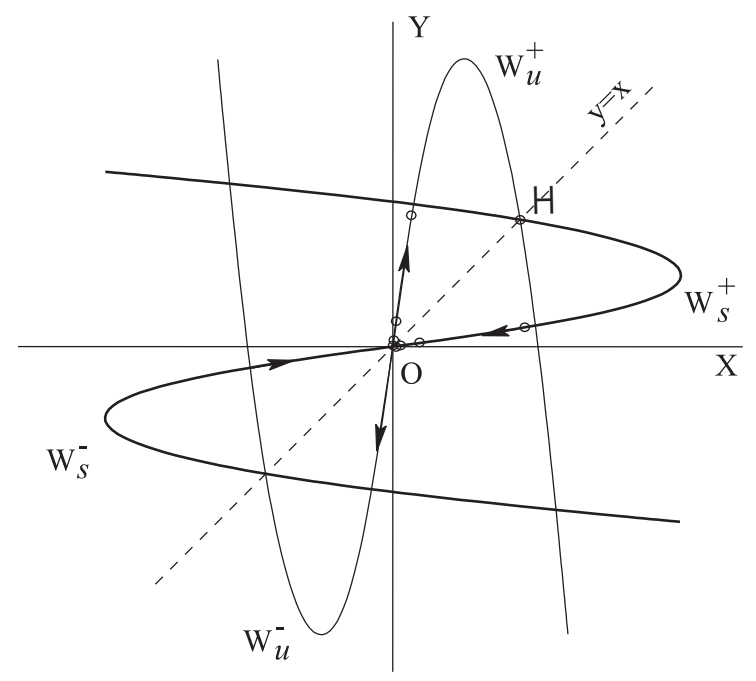

Fig. 2. An example of the branches of stable $W_{s}^{ \pm}$and unstable $W_{u}^{ \pm}$manifolds of the hyperbolic fixed point $\mathcal{O}$. The point $H$, the intersection of $W_{s}^{+}$and $W_{u}^{+}$, is a homoclinic point. The circles mark the homoclinic orbit associated with it. This orbit corresponds to the Page mode for Eq.(5).

respect to the axis $y=x$. So, any of the four branches allows one to reconstruct three others. Some analytical approximations for $W_{u, s}^{ \pm}$can be constructed using normal form analysis, see [18].

Points of intersections of $W_{s}$ and $W_{u}$ (homoclinic points) correspond to localized solutions of Eq.(5). As it was shown above, when $0<\alpha<\alpha^{*}$ all localized solutions can be coded by the sequences from $\mathcal{L}_{0}$. The homoclinic points also can be associated with these sequences. Each homoclinic point has the only matching sequence from $\mathcal{L}_{0}$, but each coding sequence from $\mathcal{L}_{0}$ corresponds to an infinite number of homoclinic points connected with each other by iterations of $T$ (homoclinic orbit). This is a natural consequence of the translational invariance of the lattice. Due to the involutions $(10),(11)$ the points of intersections of $W_{s}^{ \pm}$(and $W_{u}^{ \pm}$) with the lines $y=x$ and $y=-x$ are homoclinic points. Their codes are, correspondingly, symmetric or antisymmetric.

Let us introduce the following definitions.

Definition 3.1 We call basic points the nine points $(0,0),(-1,0),(1,0)$, $(0,1),(-1,1),(1,1),(0,-1),(-1,-1),(1,-1)$ on the plane $(x, y)$.

Definition 3.2 The spot $S_{s k}, s, k \in\{-, 0,+\}$ is a closed square on the plane $(x, y)$ with the side $2 M(\alpha)$, where $M(\alpha)$ is given by Eq.(7), centered at the basic point coded with the symbols $s$ and $k$. The symbol $s$ is the sign of abscissa of the basic point (or zero), the second one is the sign of its ordinate (or zero).

For example, $S_{+-}$is the spot which is centered in the point $(1,-1)$ (see Fig. 3). It follows from Corollary 2.1 that 


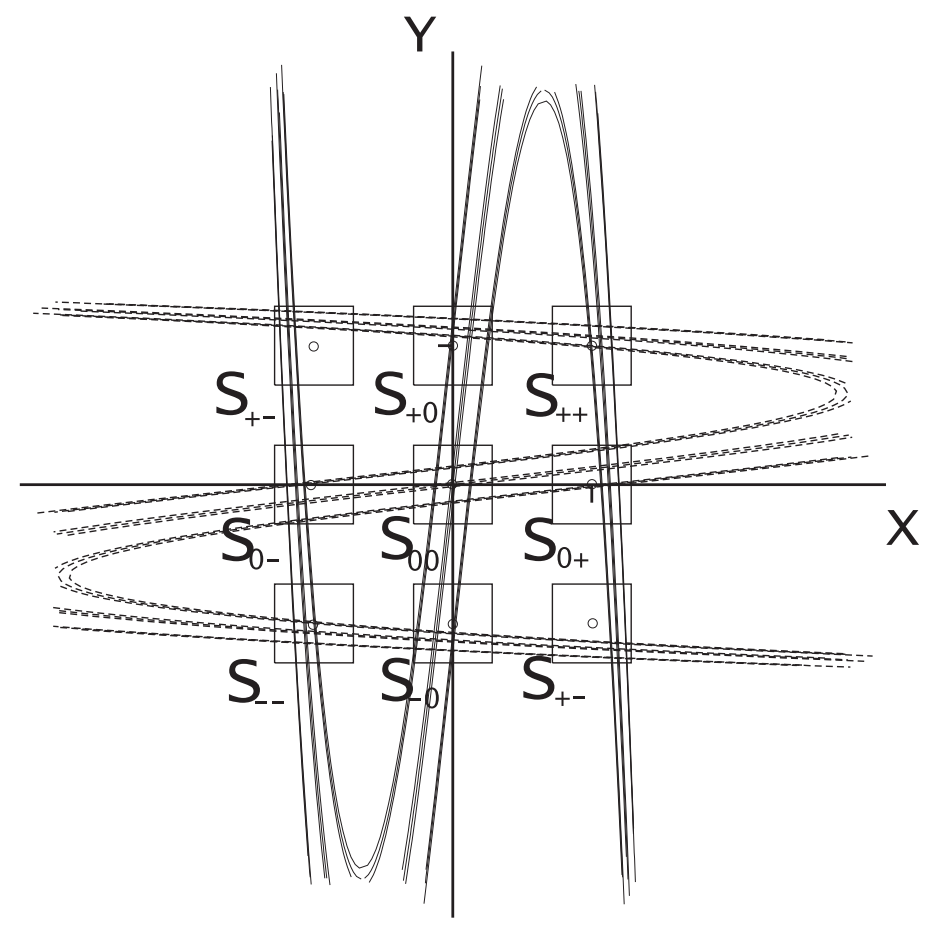

Fig. 3. The spots $S_{s k}$ and stable (dashed) and unstable (solid) manifolds of the fixed point $\mathcal{O}$. Small circles mark the basic points.

(i) for $\alpha$ small enough each homoclinic point on the plane $(x, y)$ is situated in the vicinity of one of the basic points. When $\alpha$ tends to zero the distance between the homoclinic point and the corresponding basic point also tends to zero;

(ii) all homoclinic points which tend to a basic point as $\alpha$ goes to zero can be covered by the spot centered at this basic point.

(iii) if $\alpha$ is small enough the spots corresponding to different basic points do not intersect each other.

An important fact that follows from (8) is that $T$ maps the homoclinic points from a spot $S_{s k}, s, k \in\{-, 0,+\}$ to points belonging to the three spots $S_{k-}$, $S_{k 0}, S_{k+}$ but not to any other spots.

For what follows we need the definitions of segment, initial segment and fundamental segment of $W_{u, s}^{ \pm}$(see $\left.[15,19]\right)$. They can be found in Appendix C. Also it is useful to introduce a natural parameterization on the branches $W_{s}^{ \pm}$and $W_{u}^{ \pm}$. Let the parameter $\tau$ be the length of the curve $W_{s}^{+}$measured from the fixed point $\mathcal{O}$. The point $\mathcal{O}$ corresponds to $\tau=0$. The coordinates of the points on the branch $W_{s}^{+}$will be denoted by $x_{s}^{+}(\tau)$ and $y_{s}^{+}(\tau)$. In the same manner we introduce the natural parameterization on the other branches $W_{s}^{-}, W_{u}^{+}$and $W_{u}^{-}$.

Now we prove the following auxiliary statement: 
Lemma 3.1 Let $\tilde{\gamma}=(\tilde{x}(\tau), \tilde{y}(\tau)), \tau \in\left[\tau_{1}, \tau_{2}\right]$ be a curve on the plane $(x, y)$ such that

(i) $\tilde{x}(\tau), \tilde{y}(\tau) \in C^{1}\left[\tau_{1}, \tau_{2}\right]$;

(ii) for $\tau \in\left[\tau_{1}, \tau_{2}\right]$ the curve $\tilde{\gamma}=(\tilde{x}(\tau), \tilde{y}(\tau))$ lies entirely within one spot $S_{s_{1} s_{2}}$, $s_{1,2} \in\{-, 0,+\}$

(iii) for $\tau \in\left[\tau_{1}, \tau_{2}\right]$ the functions $\tilde{x}(\tau)$ and $\tilde{y}(\tau)$ are monotonic with respect to $\tau$;

(iv) $|\dot{\tilde{y}}(\tau) / \dot{\tilde{x}}(\tau)|<1 / 2$ for all $\tau \in\left[\tau_{1}, \tau_{2}\right]$.

Let $\gamma=(x(\tau), y(\tau))$ be $T^{-1}$-image of $\tilde{\gamma}$. Then for $\alpha$ small enough the following statements are valid:

(a) for $\tau \in\left[\tau_{1}, \tau_{2}\right]$ the functions $x(\tau)$ and $y(\tau)$ are monotonic. Moreover, if $s_{2}=$ $\{0\}$ then $\operatorname{sign} \dot{x}(\tau)=\operatorname{sign} \dot{\tilde{x}}(\tau)$ and if $s_{2} \neq\{0\}$ then $\operatorname{sign} \dot{x}(\tau)=-\operatorname{sign} \dot{\tilde{x}}(\tau)$; (b) $|\dot{y}(\tau) / \dot{x}(\tau)|<1 / 2$ for all $\tau \in\left[\tau_{1}, \tau_{2}\right]$.

Proof. Consider the case when the curve $\tilde{\gamma}$ lies entirely in one of the spots $S_{s_{1} s_{2}}$ with $s_{2}=\{0\}$; the cases of $s_{2}=\{+\}$ and $s_{2}=\{-\}$ can be analyzed in the same manner. Differentiating (9) one obtains

$$
\begin{aligned}
& \dot{x}=\left(2+\frac{1}{\alpha}-\frac{3}{\alpha} \tilde{x}^{2}\right) \dot{\tilde{x}}-\dot{\tilde{y}} \\
& \dot{y}=\dot{\tilde{x}}
\end{aligned}
$$

The side of the spot $S_{s_{1} 0}$ is equal to $2 M(\alpha)$, so $\tilde{x}^{2}<4 M^{2}(\alpha)$. This implies that for $\alpha$ small enough the derivatives $\dot{x}(\tau)$ and $\dot{y}(\tau)$ do not change sign and $\operatorname{sign} \dot{x}(\tau)=\operatorname{sign} \dot{\tilde{x}}(\tau)$. Dividing Eq.(13) by Eq.(12) and taking into account (iv) one obtains (b) $\mathbf{\square}$.

Consider now the branch $W_{s}^{+}$. Let $\mathbf{z}=\left(x_{s}^{+}\left(\tau_{0}\right), y_{s}^{+}\left(\tau_{0}\right)\right)$ be the point of the first intersection of $W_{s}^{+}$with the boundary of the spot $S_{00}$. Then the initial segment $W_{s}^{+}(\mathcal{O}, \mathbf{z}]$ remains entirely within the spot $S_{00}$ and on it the following Lemma is valid:

Lemma 3.2 For $0<\tau<\tau_{0}$ and $\alpha$ small enough the functions $x_{s}^{+}(\tau)$ and $y_{s}^{+}(\tau)$ are monotone increasing and $\left|\dot{y}_{s}^{+}(\tau) / \dot{x_{s}^{+}}(\tau)\right|<1 / 2$.

Proof. First, we note that in some small neighborhood of the point $\mathcal{O}$ the map $T$ can be well approximated by its linearization. This means that there exist $\tau^{*}<\tau_{0}$ and the constants $C_{1,2}>0$ such that for $0<\tau \leq \tau^{*}$

$$
\begin{gathered}
\left|x_{s}^{+}(\tau)-\tau \cos \varphi\right|<C_{1} \tau^{2} \leq C_{1} \tau^{* 2} \\
\left|y_{s}^{+}(\tau)-\tau \sin \varphi\right|<C_{2} \tau^{2} \leq C_{2} \tau^{* 2}
\end{gathered}
$$


where $\varphi$ is the angle between a stable eigenvector of the linearized map and positive direction of the $x$-axis and

$$
\tan \varphi=\left(1+\frac{1}{2 \alpha}\right)-\sqrt{\left(1+\frac{1}{2 \alpha}\right)^{2}-1}=O(\alpha), \quad(\alpha \rightarrow 0)
$$

Let $\mathbf{z}^{*}=\left(x_{s}^{+}\left(\tau^{*}\right), y_{s}^{+}\left(\tau^{*}\right)\right)$. Then for $\alpha$ and $\tau^{*}$ small enough the initial segment $W_{s}^{+}\left(\mathcal{O}, \mathbf{z}^{*}\right]$ satisfies the conditions of Lemma 3.1. The transformation $T^{-1}$ maps $W_{s}^{+}\left(\mathcal{O}, \mathbf{z}^{*}\right]$ to another initial segment of $W_{s}^{+}$for which according to the Lemma 3.1 the conditions of the same Lemma 3.1 hold also. Repeating the procedure iteratively one concludes that for the whole initial segment $W_{s}^{+}(\mathcal{O}, \mathbf{z}]$ the statements of Lemma 3.2 are valid

Using Lemma 3.1 one can prove the following statements.

Lemma 3.3 For $\alpha$ small enough there exists a fundamental segment $B_{s}^{+} \in$ $W_{s}^{+}$such that

(i) $B_{s}^{+}$intersects the spot $S_{+0}$ and does not intersect the other spots;

(ii) the intersection $B_{s}^{+} \cap S_{+0}$ consists of one connected component;

(iii) all homoclinic points which $B_{s}^{+}$contains belong to $S_{+0}$;

(iv) if $\mathbf{h} \in B_{s}^{+}$is a homoclinic point then for any $n T^{n} \mathbf{h} \in S_{00}$.

Proof. Consider the fundamental segment $W_{s}^{+}(T \mathbf{z}, \mathbf{z}]$ where $\mathbf{z}=\left(x\left(\tau_{0}\right), y\left(\tau_{0}\right)\right)$ is defined above (see also Fig. 4). Evidently $W_{s}^{+}(T \mathbf{z}, \mathbf{z}] \subset S_{00}$ and for it the conditions of Lemma 3.1 holds. Define $B_{s}^{+} \equiv T^{-1} W_{s}^{+}(T \mathbf{z}, \mathbf{z}]=W_{s}^{+}\left(\mathbf{z}, T^{-1} \mathbf{z}\right]$ (see Fig. 4). This fundamental segment can be given in a parametric form, specifically, $B_{s}^{+}=\left(x_{s}^{+}(\tau), y_{s}^{+}(\tau)\right), \tau \in\left(\tau_{0}, \tilde{\tau}\right]$ for some $\tilde{\tau}$ and according to Lemma 3.1 for $\tau_{0}<\tau \leq \tilde{\tau}$ the coordinate $x_{s}^{+}(\tau)$ grows monotonically and $\left|\dot{y}_{s}^{+}(t) / \dot{x}_{s}^{+}(\tau)\right|<1 / 2$. Then using simple geometrical arguments one arrives to (i) and (ii). The point (iii) is a consequence of the fact that all homoclinic points belong to one of the spots. The point (iii) implies also (iv)

By symmetry one can conclude that for $\alpha$ small enough there exists a fundamental segment $B_{s}^{-} \subset W_{s}^{-}$such that all homoclinic points which belong to $B_{s}^{-}$lie in $S_{-0}$. Analogously, there exist the fundamental segments $B_{u}^{+} \subset W_{u}^{+}$ and $B_{u}^{-} \subset W_{u}^{-}$, such that all homoclinic points which belong to $B_{u}^{ \pm}$lie in $S_{0 \pm}$. We call $B_{s}^{ \pm}$and $B_{u}^{ \pm}$the basic fundamental segments.

The following statements are valid

Lemma 3.4 For $\alpha$ small enough and for any $n \in \mathbb{Z}$ there exist the following one-to-one correspondences:

(i) between the homoclinic points which belong to the fundamental segment $T^{n} B_{s}^{+}$and the set of the sequences from $\mathcal{L}_{0}$ which have the codes with last 


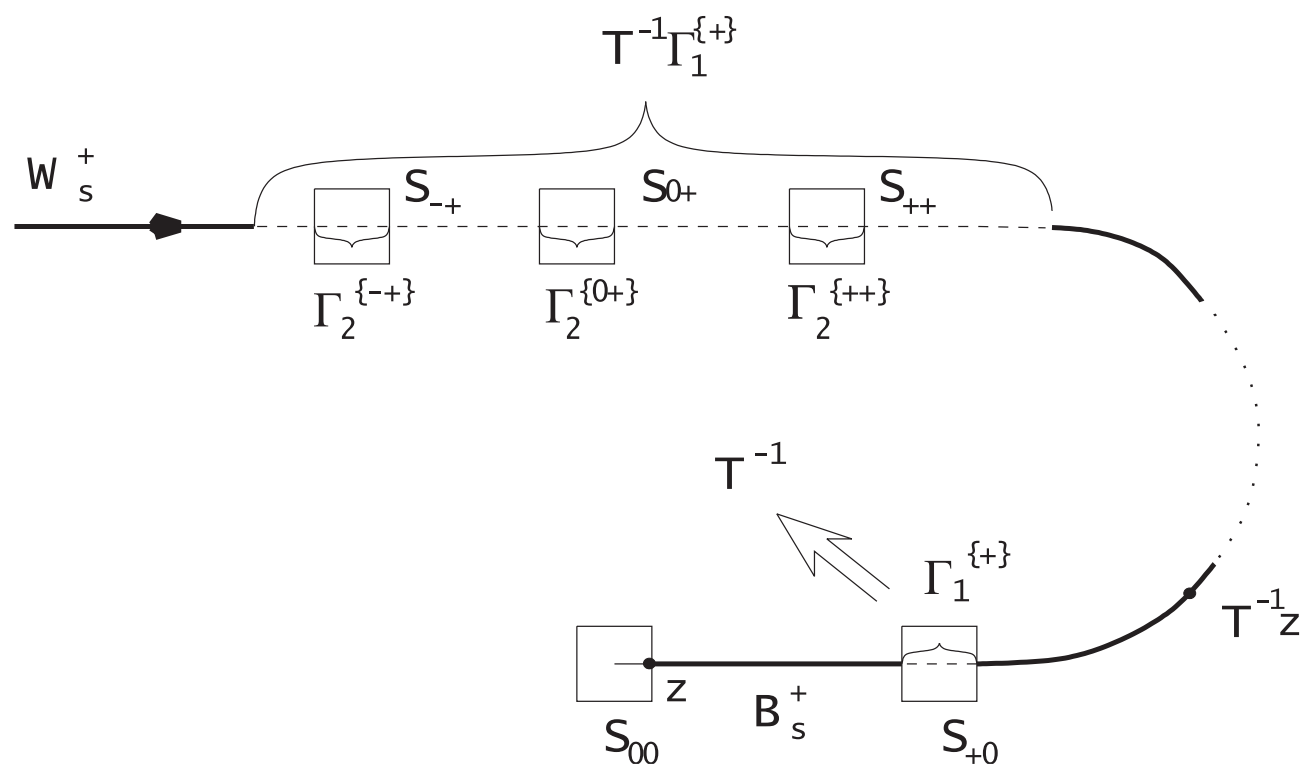

Fig. 4. A basic fundamental segment $B_{s}^{+}=W_{s}^{+}\left(\mathbf{z}, T^{-1} \mathbf{z}\right]$. All homoclinic points belonging to $B_{s}^{+}$also belong to $\Gamma_{1}^{\{+\}}=S_{+0} \cap B_{s}^{+} . T^{-1}$-images of these points lie in $\Gamma_{2}^{\{-+\}}, \Gamma_{2}^{\{0+\}}$ and $\Gamma_{2}^{\{++\}}$. The notations are defined in the text.

$$
\text { symbol "+"; }
$$

(ii) between the homoclinic points which belong to the fundamental segment $T^{n} B_{s}^{-}$and the set of the sequences from $\mathcal{L}_{0}$ which have the codes with last symbol "-"

(iii) between the homoclinic points which belong to the fundamental segment $T^{n} B_{u}^{+}$and the set of the sequences from $\mathcal{L}_{0}$ which have the codes with first symbol " + ";

(iv) between the homoclinic points which belong to the fundamental segment $T^{n} B_{u}^{-}$and the set of the sequences from $\mathcal{L}_{0}$ which have the codes with first symbol "-".

Proof. As an illustration let us consider the point (i) and $n=0$ (the basic fundamental segment $B_{s}^{+}$itself); all other cases can be treated similarly. Let $h$ be a homoclinic point from $B_{s}^{+}$. Consider the corresponding solution of Eq.(5). Its coding sequence from $\mathcal{L}_{0}$ contains the infinite block $(+, 0,0,0 \ldots)$, so the code end up with the symbol " + ". From the other side, consider a sequence $L \in \mathcal{L}_{0}$ which end up with the block $(+, 0,0,0 \ldots)$. If $\alpha$ is small enough, there exists a solution of Eq.(5) which corresponds to $L$. On the plane $(x, y)$ its image is a sequence of homoclinic points $h_{k}, k \in \mathbb{Z}, T h_{k}=h_{k+1}$. According to the coding sequence, there exists a number $k_{0}$, such that for $k>k_{0} h_{k} \in S_{00}$ and $h_{k_{0}} \in S_{0+}$. Then $h_{k_{0}} \in B_{s}^{+}$by construction of $B_{s}^{+}$in Lemma $3.3 \mathbf{a}$.

In what follows we will label homoclinic points by their codes saying, for example, "homoclinic point $\{-+-\}$ ". This labeling is well-defined within one fundamental segment: two points which belong to the same fundamental 
segment definitely have different codes. In what follows if the fundamental segment is not specified, this means that the homoclinic point under consideration belongs to $B_{s}^{ \pm}$.

\subsection{Ordering on $B_{s}^{ \pm}$}

We say that $\mathbf{a}>_{s} \mathbf{b}\left(\mathbf{a}<_{s} \mathbf{b}\right)$ on the branch $W_{s}^{ \pm}$if $\mathbf{b}$ lies closer (farther) on $W_{u}^{ \pm}$to the hyperbolic fixed point $\mathcal{O}$ than a. The ordering on $W_{u}^{ \pm}$is defined in the same manner.

We denote by $\left\{\left\{\xi_{1}\right\}\left\{\xi_{2}\right\}\right\}$ the word composed by two words $\left\{\xi_{1}\right\}$ and $\left\{\xi_{2}\right\}$. For example, if $\left\{\xi_{1}\right\}=\{++\}$ and $\left\{\xi_{2}\right\}=\{--\}$ then $\left\{\left\{\xi_{1}\right\}\left\{\xi_{2}\right\}\right\}=\{++--\}$.

Then the following Theorem establishes the ordering on $B_{s}^{+}$:

Theorem 3.1 Let $\alpha$ be small enough and $\{\xi\}$ be a word of arbitrary but finite length. Then if the total number of symbols $\{+\}$ and $\{-\}$ in $\{\xi\}$ is odd then for any three words $\left\{\eta_{1}\right\},\left\{\eta_{2}\right\},\left\{\eta_{3}\right\}$ the homoclinic points on $B_{s}^{+}$are ordered as follows

$$
\left\{\left\{\eta_{1}\right\}-\{\xi\}+\right\}<_{s}\left\{\left\{\eta_{2}\right\} 0\{\xi\}+\right\}<_{s}\left\{\left\{\eta_{3}\right\}+\{\xi\}+\right\}
$$

and if $\{\xi\}$ contains even number of the symbols $\{+\}$ and $\{-\}$ then

$$
\left\{\left\{\eta_{1}\right\}+\{\xi\}+\right\}<_{s}\left\{\left\{\eta_{2}\right\} 0\{\xi\}+\right\}<_{s}\left\{\left\{\eta_{3}\right\}-\{\xi\}+\right\} .
$$

Proof. First, let us verify this statement for the words $\{\xi\}$ of length 1 . Define $\Gamma_{0}^{\{+\}} \equiv W_{s}^{+}(\mathcal{O}, \mathbf{z}]$ (where $\mathbf{z}$ is defined in subsection 3.1) and $\Gamma_{1}^{\{+\}} \equiv B_{s}^{+} \cap S_{+0}$ (see Fig. 4). Evidently, $T \Gamma_{1}^{\{+\}} \subset \Gamma_{0}^{\{+\}}$. According to Lemma $3.3 \Gamma_{1}^{\{+\}}$consists of a single connected component and there exist $\tau_{1}^{+}$and $\tau_{2}^{+}$such that $\Gamma_{1}^{\{+\}}=$ $\left(x_{s}^{+}(\tau), y_{s}^{+}(\tau)\right)$ for $\tau_{1}^{+}<\tau<\tau_{2}^{+}$. Consider now $T^{-1}$-image of $\Gamma_{1}^{\{+\}}$. According to Lemma $3.1 T^{-1} \Gamma_{1}^{\{+\}}=\left(x_{s}^{+}(\tau), y_{s}^{+}(\tau)\right)$ where $\tau$ varies within some interval $\tilde{\tau}_{1}<\tau<\tilde{\tau}_{2}$, the function $x_{s}^{+}(\tau)$ monotonically decreases within this interval and

$$
\left|\dot{y}_{s}^{+}(\tau) / \dot{x}_{s}^{+}(\tau)\right|<1 / 2, \quad \tau_{1}^{+}<\tau<\tau_{2}^{+}
$$

The homoclinic points which lie in $\Gamma_{1}^{\{+\}}$are $T$-images of homoclinic points which belong to fundamental segment $T^{-1} B_{s}^{+}$. They are situated in the spots $S_{++}, S_{0+}$ and $S_{-+}$. So, $T^{-1} \Gamma_{1}^{\{+\}}$intersects these three spots. Since $x_{s}^{+}$decreases monotonically on $T^{-1} \Gamma_{1}^{\{+\}}$the intersections are in the order given above (see 
Fig. 4). It follows from (16) that $T^{-1} \Gamma_{1}^{\{+\}}$does not intersect any other spot. Denote

$$
\begin{aligned}
\Gamma_{2}^{\{++\}} & =T^{-1} \Gamma_{1}^{\{+\}} \cap S_{++}, \\
\Gamma_{2}^{\{0+\}} & =T^{-1} \Gamma_{1}^{\{+\}} \cap S_{0+}, \\
\Gamma_{2}^{\{-+\}} & =T^{-1} \Gamma_{1}^{\{+\}} \cap S_{-+} .
\end{aligned}
$$

Each of $\Gamma_{2}^{\{++\}}, \Gamma_{2}^{\{0+\}}$ and $\Gamma_{2}^{\{-+\}}$consists of one connected component. On the fundamental segment $T^{-1} B_{s}^{+}$the arc $\Gamma_{2}^{\{++\}}$includes all homoclinic points corresponding to the codes $\left\{\left\{\eta_{1}\right\}++\right\}$, the arc $\Gamma_{2}^{\{0+\}}$ includes all homoclinic points corresponding to the codes $\left\{\left\{\eta_{2}\right\} 0+\right\}$ and the arc $\Gamma_{2}^{\{-+\}}$- all homoclinic points corresponding to the codes $\left\{\left\{\eta_{3}\right\}-+\right\}$ (here $\eta_{1}, \eta_{2}$ and $\eta_{3}$ are arbitrary words). This implies that on $B_{s}^{+}$

$$
\left\{\left\{\eta_{1}\right\}++\right\}<_{s}\left\{\left\{\eta_{2}\right\} 0+\right\}<_{s}\left\{\left\{\eta_{3}\right\}-+\right\}
$$

In general case we denote the corresponding segments by recurrence relations

$$
\begin{aligned}
\Gamma_{n}^{\{+k\{\zeta\}+\}} & =T^{-1} \Gamma_{n-1}^{\{k\{\zeta\}+\}} \cap S_{+k}, \\
\Gamma_{n}^{\{0 k\{\zeta\}+\}} & =T^{-1} \Gamma_{n-1}^{\{k\{\zeta\}+\}} \cap S_{0 k}, \\
\Gamma_{n}^{\{-k\{\zeta\}+\}} & =T^{-1} \Gamma_{n-1}^{\{k\{\zeta\}+\}} \cap S_{-k},
\end{aligned}
$$

where $\{\zeta\}$ runs all the words of length $(n-2)$ and $k \in\{-, 0,+\}$. Arcs $\Gamma_{n}^{\{+k\{\zeta\}+\}}, \Gamma_{n}^{\{0 k\{\zeta\}+\}}$ and $\Gamma_{n}^{\{-k\{\zeta\}+\}}$ belong to fundamental segment $T^{-(n-1)} B_{s}^{+}$. Similarly to the considered case using Lemma 3.1 one can conclude that

(a) $T^{-1} \Gamma_{n-1}^{\{k\{\zeta\}+\}}$ intersects the three spots $S_{+k}, S_{0 k}$ and $S_{-k}$ and does not intersect the other spots;

(b) Each of $\Gamma_{n}^{\{+k\{\zeta\}+\}}, \Gamma_{n}^{\{0 k\{\zeta\}+\}}$ and $\Gamma_{n}^{\{-k\{\zeta\}+\}}$ consists of only one connected component, so each of these arcs can be parameterized in the form $\left(x_{s}^{+}(\tau), y_{s}^{+}(\tau)\right)$ at appropriate interval of $\tau$.

(c) The order in which $\Gamma_{n}^{\{+k\{\zeta\}+\}}, \Gamma_{n}^{\{0 k\{\zeta\}+\}}$ and $\Gamma_{n}^{\{-k\{\zeta\}+\}}$ appear on $T^{-(n-1)} B_{s}^{+}$ coincides with the order in which $\Gamma_{n-1}^{\{+\{\zeta\}+\}}, \Gamma_{n-1}^{\{0\{\zeta\}+\}}$ and $\Gamma_{n-1}^{\{-\{\zeta\}+\}}$ appear on $T^{-(n-2)} B_{s}^{+}$if $k=\{0\}$ and is opposite to it if $k=\{+\}$ or $k=\{-\}$.

A schematic picture of relations between the $\operatorname{arcs} \Gamma_{n}^{\{\{\xi\}+\}}$ is shown in the Fig. 5. Taking into account that the homoclinic points which belong to $\Gamma_{n}^{\{\{\xi\}+\}}$ correspond to the codes $\{\{\eta\}\{\xi\}+\}$ where $\eta$ is an arbitrary word we obtain the statement of the Theorem 


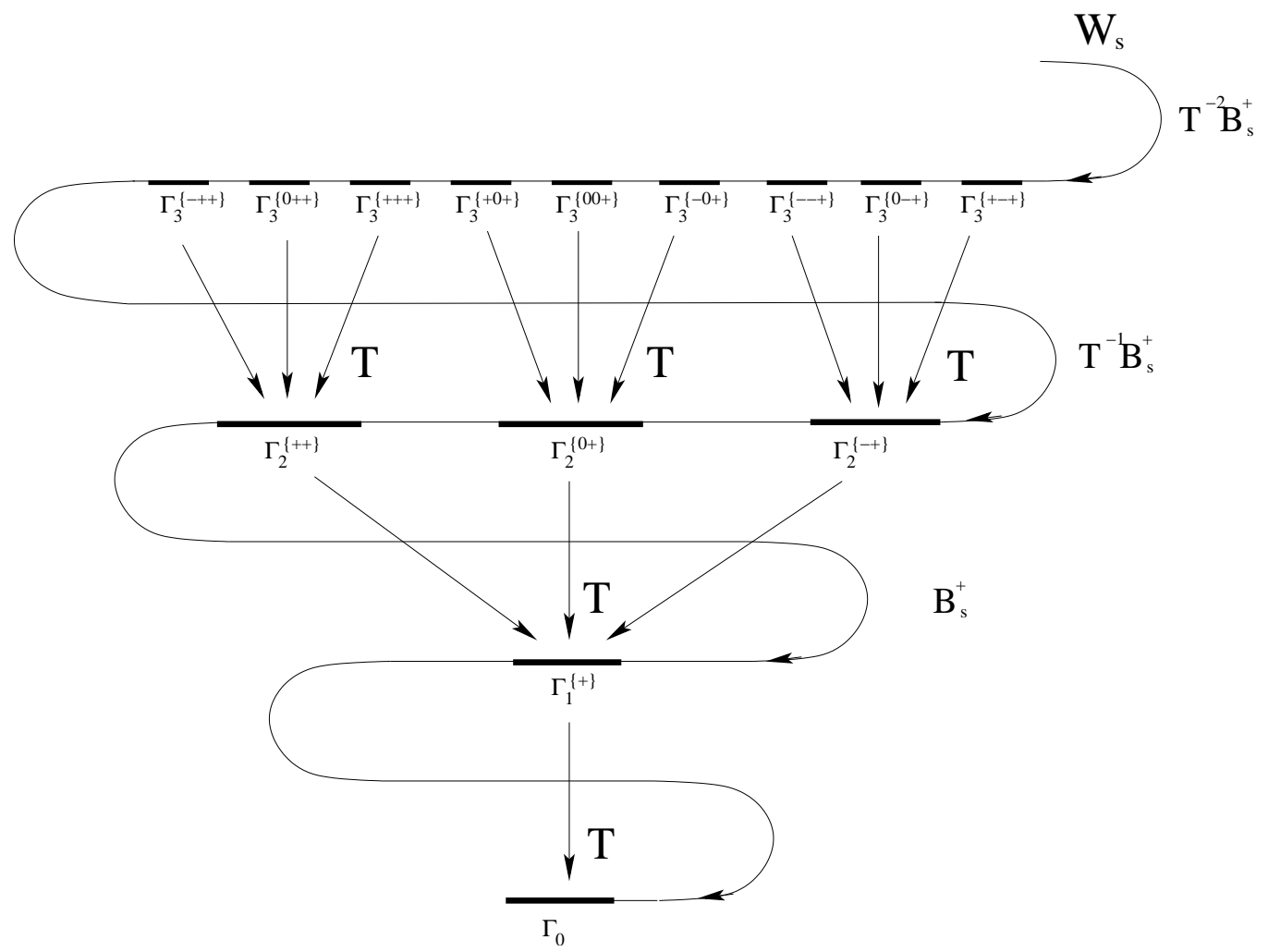

Fig. 5. A schematic picture of relations between the arcs $\Gamma_{n}^{\{\{\xi\}+\}}$

Remark. Theorem 3.1 gives a simple recurrence algorithm allowing one to establish the proper ordering of homoclinic points with codes of lengths smaller than some prescribed length on $B_{s}^{+}$. It consists in the following steps:

Step 1. To write the starting codes $\{++\},\{0+\},\{-+\}$ (exactly in this order) and the arrow pointed from $\{-+\}$ to $\{++\}$.

Step $n$. Let the codes for $(n-1)$-st step be just written.

Then one has

1. To draw arrows corresponding to each of the codes such that:

(a) the extreme left arrow is pointed in opposite direction to the extreme left arrow on the previous step;

(b) the neighboring arrows have opposite directions.

2. To generate new string of codes replacing $\{\xi\}$ by the three codes $\{-\{\xi\}\}$, $\{0\{\xi\}\},\{+\{\xi\}\}$ if the arrow corresponding to $\{\xi\}$ is pointed to the right and $\{+\{\xi\}\},\{0\{\xi\}\},\{-\{\xi\}\}$ if the arrow is pointed to the left (see Fig.6).

In Fig. 6 the largest arrow corresponds to the first step of the algorithm, three smaller ones correspond to the Step 2, and nine short arrows correspond to the 


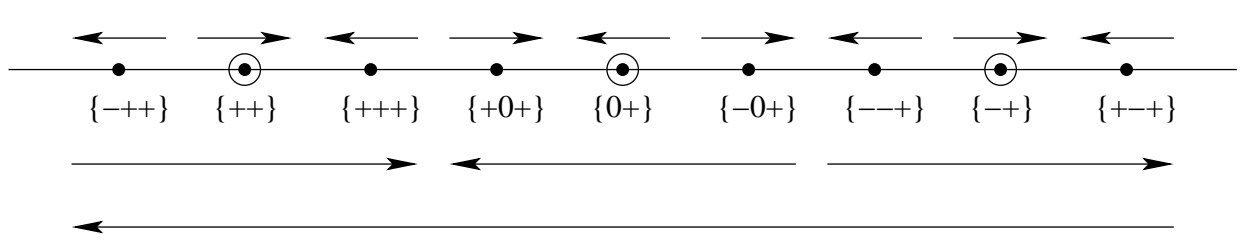

Fig. 6. How to place the homoclinic points on $B_{s}^{+}$in the proper order (illustration to the algorithm).

third step. As a result, the picture shows the mutual position of the homoclinic points with the code length smaller than 3 on $B_{s}^{+}$.

The same algorithm allows one to order homoclinic points on other basic fundamental segments $B_{s}^{-}, B_{u}^{+}$and $B_{u}^{-}$. By comparing orders of homoclinic points on both stable and unstable manifolds, it is possible to represent schematically how $W_{u}^{+}$and $W_{u}^{-}$intersect $W_{s}^{+}$(see Fig.7). Also it is possible to describe a structure of homoclinic points which are situated in a neighborhood of a homoclinic point $\{\xi\}$ (see Fig.8).

\section{Homoclinic bifurcations}

Theorem 2.1 states that for the interval $0<\alpha<\alpha^{*}$ all localized solutions of Eq.(5) are in one-to-one correspondence with the set of all sequences from $\mathcal{L}_{0}$. If $\alpha$ exceeds the critical value $\alpha^{*}$ this does not take place any more and solutions can appear or disappear as a result of some bifurcations. Since the localized solutions correspond to the points of intersections of the stable $W_{s}$ and the unstable $W_{u}$ manifolds of the fixed point $\mathcal{O}$, these bifurcations can be found numerically by observing how the forms of $W_{s}$ and $W_{u}$ change as $\alpha$ grows. In such a way, each of the solutions of Eq.(5) which exists for $0<\alpha<\alpha^{*}$ can be continued until some value of $\alpha>\alpha^{*}$ where it dies in some bifurcation. It is convenient to label this solution with the same code as it has in the anticontinuous limit. In this sense, the coding of solutions used for $0<\alpha<\alpha^{*}$ can be used for $\alpha>\alpha^{*}$ also. A problem can occur, however, if some new solutions appear since they have no counterparts in the set of the coding sequences.

In order to study possible bifurcations we used a numerical procedure which allows one to construct initial segments of the manifolds $W_{u}$ and $W_{s}$, to find their points of intersection, and to identify them with possible codes from $\mathcal{L}_{0}$. Using this program we observed that the deformation of the manifolds $W_{u}$ and $W_{s}$ as $\alpha$ grows is quite monotonic. This results in numerous bifurcations of death of homoclinic points. However, we did not observe any bifurcation of birth of new homoclinic point. This allows us to formulate the following conjecture. 


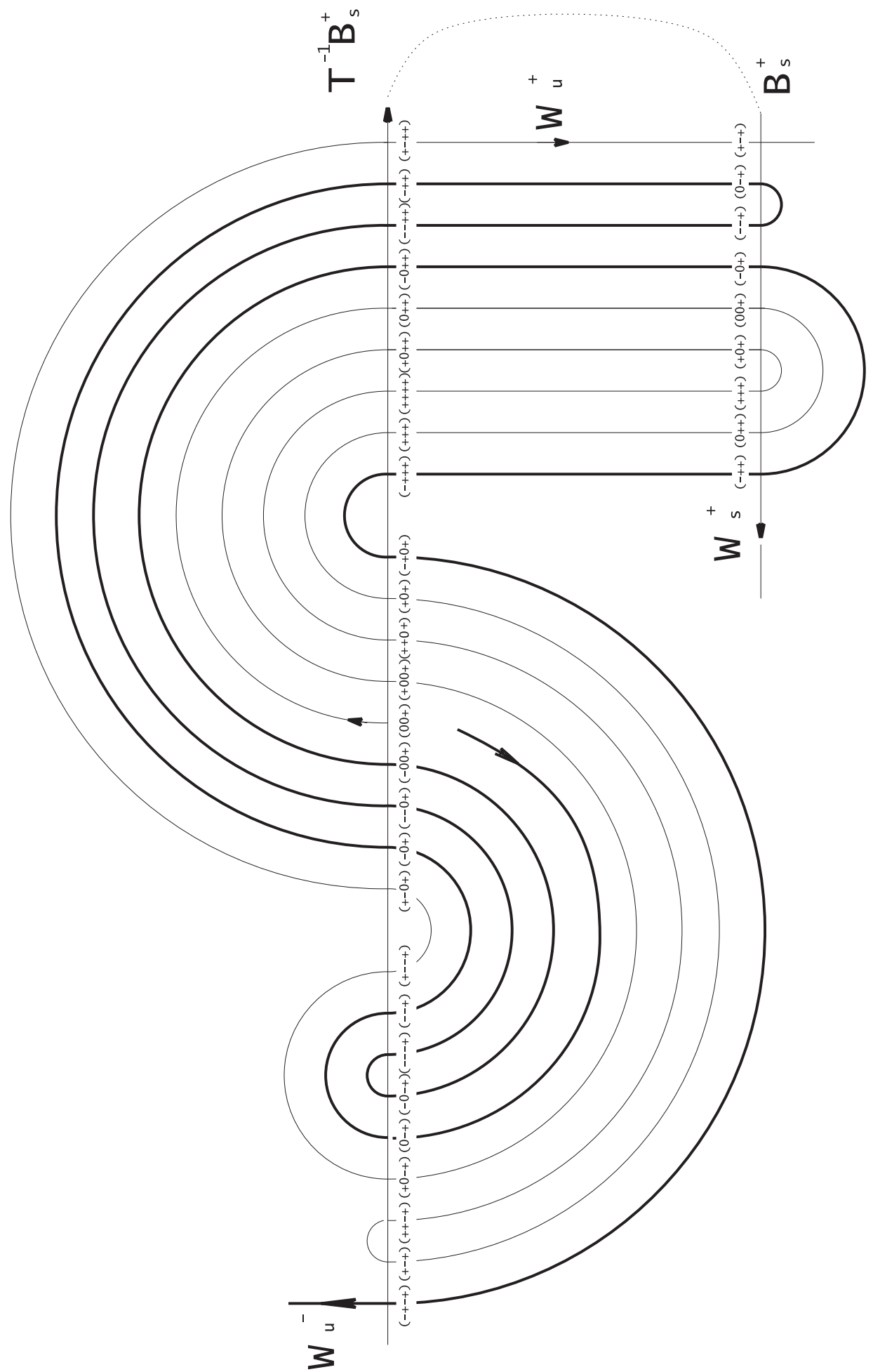

Fig. 7. Schematic representation of the intersections of $W_{u}^{+}$(thin line) and $W_{u}^{-}$ (bold line) with the branch $W_{s}^{+}$. 


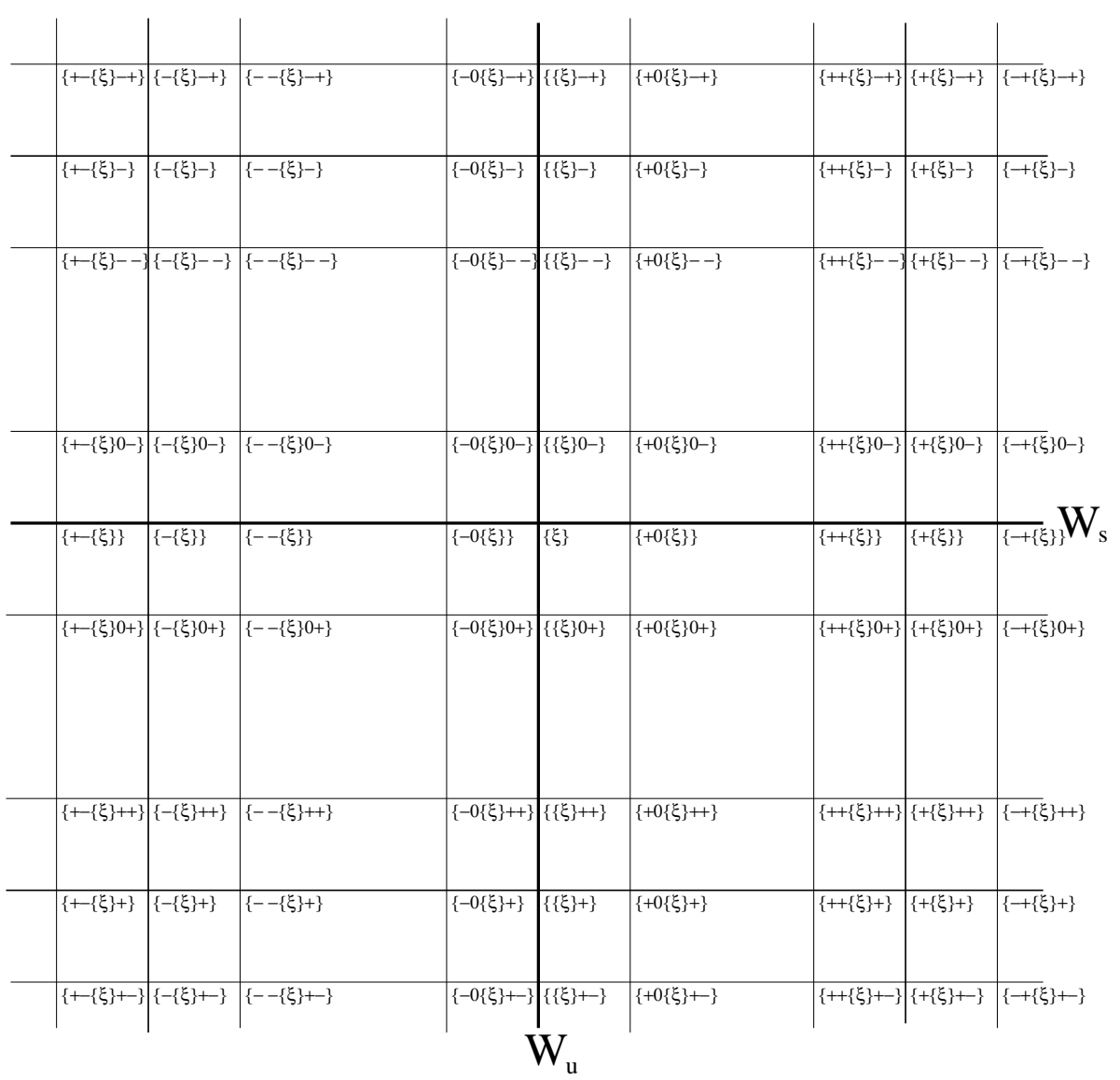

Fig. 8. The homoclinic points in a neighborhood of the homoclinic point with the code $\{\xi\}$.

Conjecture 4.1 As a grows no bifurcations of birth of new solutions occur.

This conjecture is similar to "No-bubbles-conjecture" of [15] for Hénon map. In view of this conjecture the problem is reduced to the description of the bifurcations of death of solutions which can be identified with their codes in the anticontinuous limit.

Let us introduce the following notations. Let $\{\xi\}$ be a finite word. We denote by $\{\bar{\xi}\}$ the word formed by the symbols of $\{\xi\}$ taken in inverse order. Also we denote $\{\tilde{\xi}\}$ the word obtained from $\{\xi\}$ by replacing the symbols " + " by " -" and vice versa. We say that $\{\xi\}$ is symmetric if $\{\xi\}=\{\bar{\xi}\}$ and antisymmetric if $\{\tilde{\xi}\}=\{\bar{\xi}\}$. Also we call symmetric (antisymmetric) the homoclinic point coded with symmetric (antisymmetric) word $\{\xi\}$. In the same manner we define symmetric (antisymmetric) solutions of Eq.(5). Then the two types of homoclinic bifurcations are generic in our case:

$\underline{\text { Saddle-node bifurcation. In a point of the saddle-node bifurcation } \alpha=\alpha_{S N}}$ two homoclinic points which exist for $\alpha<\alpha_{S N}$, merge at $\alpha=\alpha_{S N}$, and do not exist for $\alpha>\alpha_{S N}$. It is clear that if two homoclinic points with codes $\left\{\xi_{1}\right\}$ 

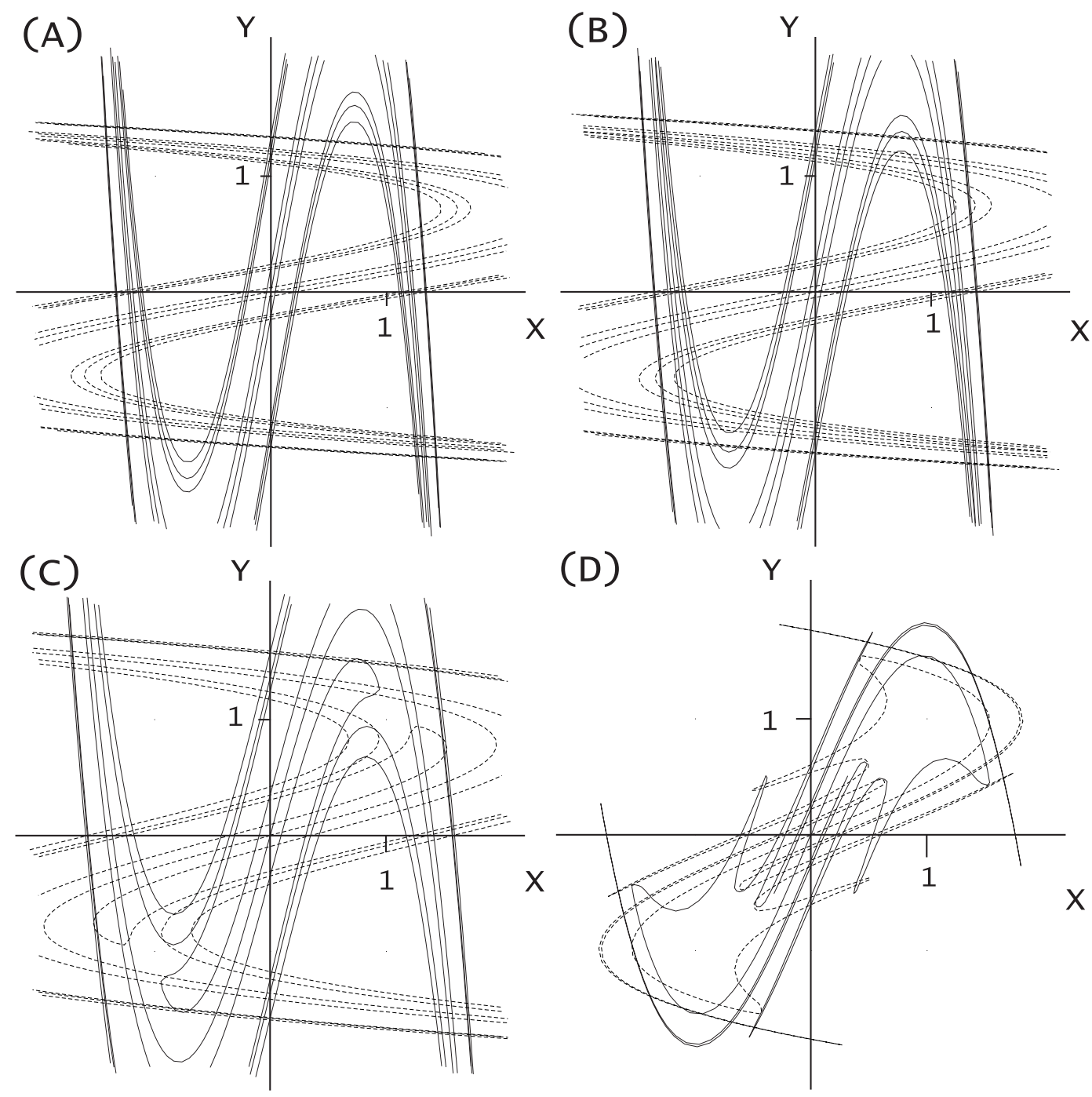

Fig. 9. The stable (dashed line) and the unstable (solid line) manifolds of the fixed point $\mathcal{O}$. (a) $\alpha \approx 0.2632$, $(\omega=3.8)$. In this case $\alpha<\alpha^{*}$ and the structure of the intersections of the stable and unstable manifolds is the same as in the anticontinuous limit. (b) $\alpha \approx 0.3125$, ( $\omega=3.2$ ), some homoclinic points disappeared; (c) $\alpha \approx 0.5$, $(\omega=2) ;(\mathrm{d}) \alpha \approx 1.25,(\omega=0.8)$.

and $\left\{\xi_{2}\right\}$ undergo the saddle-node bifurcation, then the pairs of homoclinic points $\left(\left\{\tilde{\xi}_{1}\right\},\left\{\tilde{\xi}_{2}\right\}\right),\left(\left\{\bar{\xi}_{1}, \bar{\xi}_{2}\right\}\right)$, and $\left(\left\{\tilde{\bar{\xi}}_{1}\right\},\left\{\tilde{\bar{\xi}}_{2}\right\}\right)$ also undergo the saddle-node bifurcation at the same $\alpha=\alpha_{S N}$.

Pitchfork bifurcation. In a point of the pitchfork bifurcation $\alpha=\alpha_{P}$ two homoclinic points with codes $\left\{\xi_{1}\right\}$ and $\left\{\xi_{2}\right\}$ related by the symmetry $\left\{\xi_{1}\right\}=$ $\left\{\bar{\xi}_{2}\right\}$ or antisymmetry $\left\{\xi_{1}\right\}=\left\{\tilde{\bar{\xi}}_{2}\right\}$ die by merging with a third homoclinic point $\left\{\xi_{3}\right\}$. The code $\left\{\xi_{3}\right\}$ of the resulting homoclinic point is symmetric if $\left\{\xi_{1}\right\}=\left\{\bar{\xi}_{2}\right\}$ and antisymmetric if $\left\{\xi_{1}\right\}=\left\{\tilde{\bar{\xi}}_{2}\right\}$. This bifurcation is generic since the dynamical system possesses involutions. 

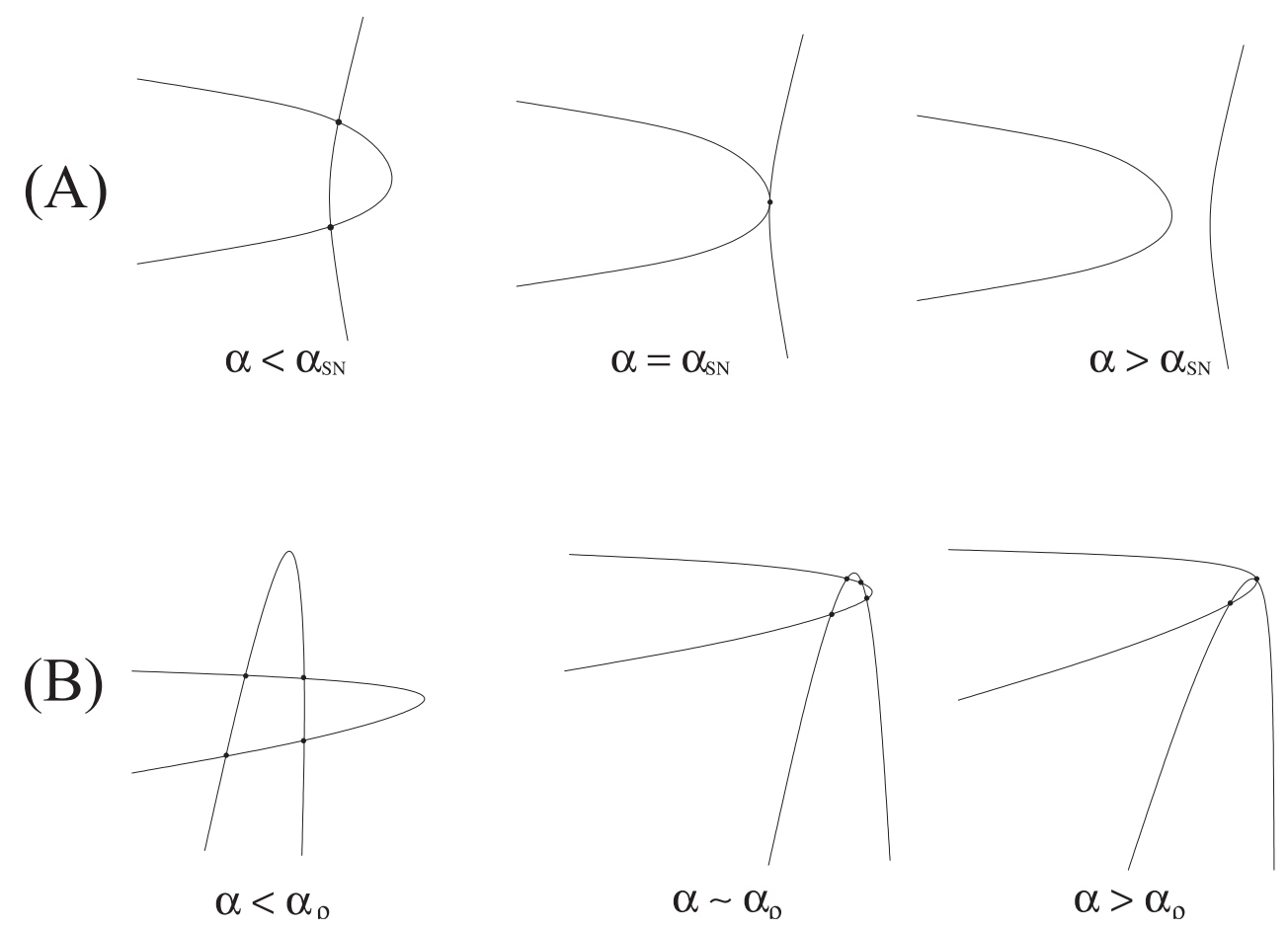

Fig. 10. Saddle-node (a) and pitchfork (b) bifurcations illustrated in terms of intersections of lobes of stable and unstable manifolds.

The both types of bifurcations can be illustrated schematically considering a generic intersection of the lobes of $W_{s}$ and $W_{u}$ (see Fig.10). It follows from Fig.10 that a pitchfork bifurcation is typically followed by a saddle-node bifurcation. In this saddle-node bifurcation the symmetric (antisymmetric) homoclinic point (the "output" of the pichfork bifurcation) merges with one more homoclinic point of the same symmetry. We checked numerically (see below) that for $\alpha>\alpha^{*}$ the both types of the bifurcations take place.

Following [15] we call homoclinic points $\mathbf{a}$ and $\mathbf{b}$ double neighbors if there are no other homoclinic points on the segments $W_{u}(\mathbf{a}, \mathbf{b})$ and $W_{s}(\mathbf{a}, \mathbf{b})$. Homoclinic points cannot undergo bifurcation unless they are double neighbors. This observation implies that

Lemma 4.1 The codes of bifurcating homoclinic points (solutions) must start and end with the same symbol.

Proof. This statement immediately follows from the fact that the homoclinic points with codes starting with "+" lie on the branch $W_{u}^{+}$whereas ones with codes starting with " - " belong to $W_{u}^{-}$. So, the homoclinic points with different first symbol of the code belong to different branches and cannot be neighbors. The same arguments can be used for the last symbol of the code

Let us now turn to the analysis, what happens on the boundary of the interval 
$0<\alpha<\alpha^{*}$

Lemma 4.2 The value $\alpha=\alpha^{*}$ is not a value of saddle-node or pitchfork bifurcation.

Proof. It follows from Theorem 3.1 that for $0<\alpha<\alpha^{*}$ there exist infinitely many homoclinic points between any pair of homoclinic points which belongs to $B_{s}^{ \pm}$. This implies that there are no double neighbors at $B_{s}^{ \pm}$for $\alpha=\alpha^{*} \mathbf{\square}$.

Let $\{\xi\}$ be arbitrary finite word. Then we define the word

$$
\{\xi\}^{n} \equiv\{\underbrace{\{\xi\}\{\xi\} \ldots\{\xi\}}_{\mathrm{n} \text { times }}\}
$$

and its limit case

$$
\{\xi\}^{\infty} \equiv\{\ldots\{\xi\}\{\xi\} \ldots\{\xi\} \ldots\}
$$

The results of numerical investigation allow us to formulate the following statement:

Statement 4.1 The value $\alpha^{*} \approx 0.28958$ is a limit value of a sequence of points of homoclinic saddle-node bifurcations. Bifurcating pairs of homoclinic points have the codes $\left\{+\{-+\}^{n}+\{+-\}^{n}\right\}$ and $\left\{+\{-+\}^{n} 0\{+-\}^{n}\right\}, n-$ positive integer. This value $\alpha^{*}$ also can be understood (and found numerically in practice) as a point of saddle-node bifurcation of the solution of (5) coded by $\left\{\{-+\}^{\infty}+\{+-\}^{\infty}\right\}$ which is double asymptotic to the periodic solution $\{+-\}^{\infty}$.

We comment Statement 4.1 graphically as follows. In Fig. 11 there are plotted the parts of stable and unstable manifolds which pass through the area of the bifurcations. There are two rectangles marked as (A) and (B) in Fig. 11. The coding of homoclinic points within these rectangles can be understood from Fig.8. Specifically, in the case of the rectangle (A) one should take $\xi=$ $\{++\}$ and reflect Fig. 8 with respect to central vertical line; in the case of the rectangle $(\mathrm{B})$ one should take $\xi=\{+0\}$ and rotate the Fig. 8 by 180 degrees. As $\alpha$ grows the points from the right lower corner of $(\mathrm{A})$ tend to the corresponding points from right upper corner of $(\mathrm{B})$. Up to the lines shown in Fig. 8 this corresponds to the saddle-node bifurcation of homoclinic points with codes $\{+-+++-\}$ and $\{+-+0+-\}$. Completing the picture of Fig. 8 i.e. adding more horizontal and vertical lines we found other codes of 


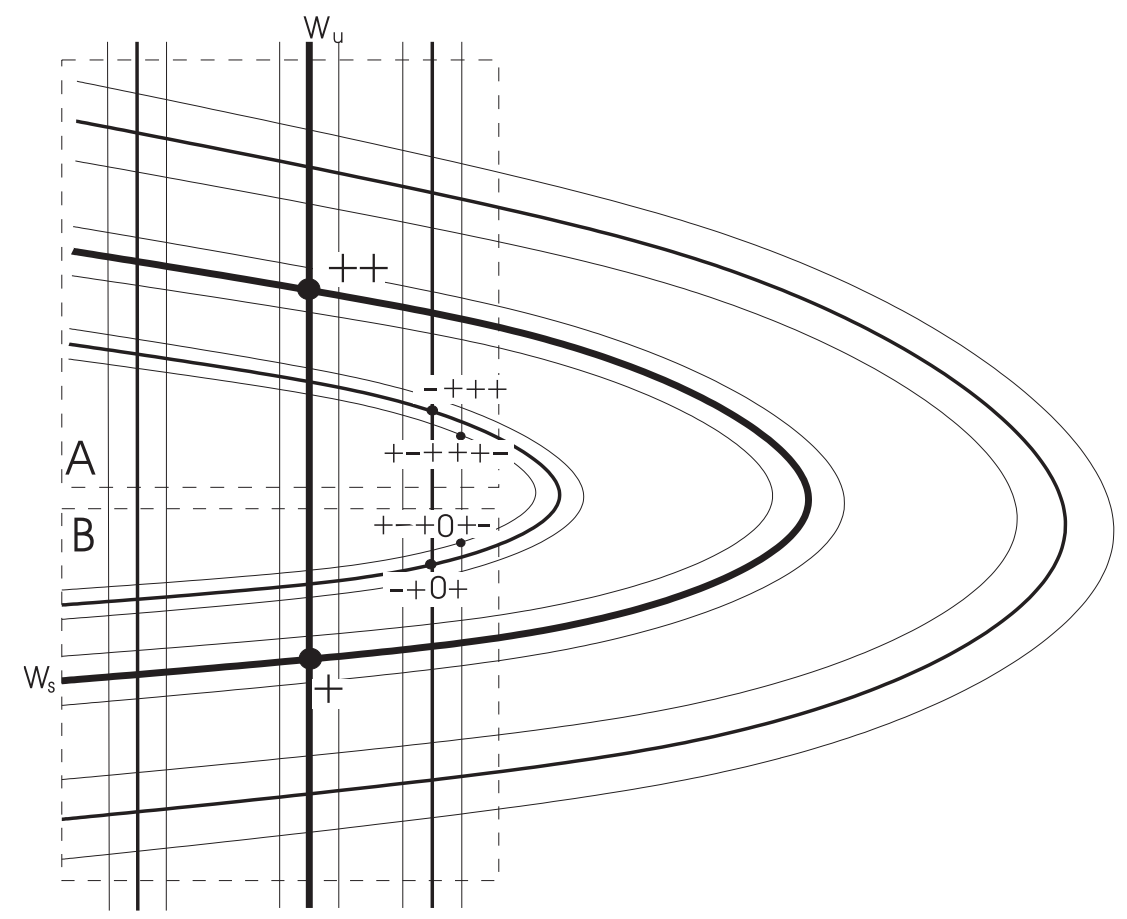

Fig. 11. The parts of the stable and unstable manifolds which pass through the area of the bifurcations, $\alpha \sim \alpha^{*}$. The bolder lines correspond to the earlier passages of the stable and unstable manifolds through the area.

bifurcating homoclinic points:

$$
\begin{array}{ll}
\{-+-+++-+\} & \Longleftrightarrow\{-+-+0+-+\}, \\
\{+-+-+++-+-\} & \Longleftrightarrow\{+-+-+0+-+-\}, \\
\{-+-+-+++-+-+\} & \Longleftrightarrow\{-+-+-+0+-+-+\},
\end{array}
$$

Bifurcations of words with greater lengths occur at smaller values of $\alpha$ than the ones of words with smaller lengths of code. The codes of the bifurcating homoclinic points approach the infinite sequences

$$
\left\{\{-+\}^{\infty}+\{+-\}^{\infty}\right\} \text { and } \quad\left\{\{-+\}^{\infty} 0\{+-\}^{\infty}\right\}
$$

These sequences correspond to the orbits homoclinic to periodic orbit with the code $\{+-\}^{\infty}$. The bifurcations of these orbits can be found by studying stable and unstable manifolds of the fixed point $\{+\}^{\infty}$ of the squared map $T^{2}$. This analysis shows that (18) undergo saddle-node bifurcation at $\alpha^{*} \approx 0.28958$. Also it was confirmed numerically that the points of the bifurcations of pairs (17) approach this value as the length of the corresponding codes grows. 


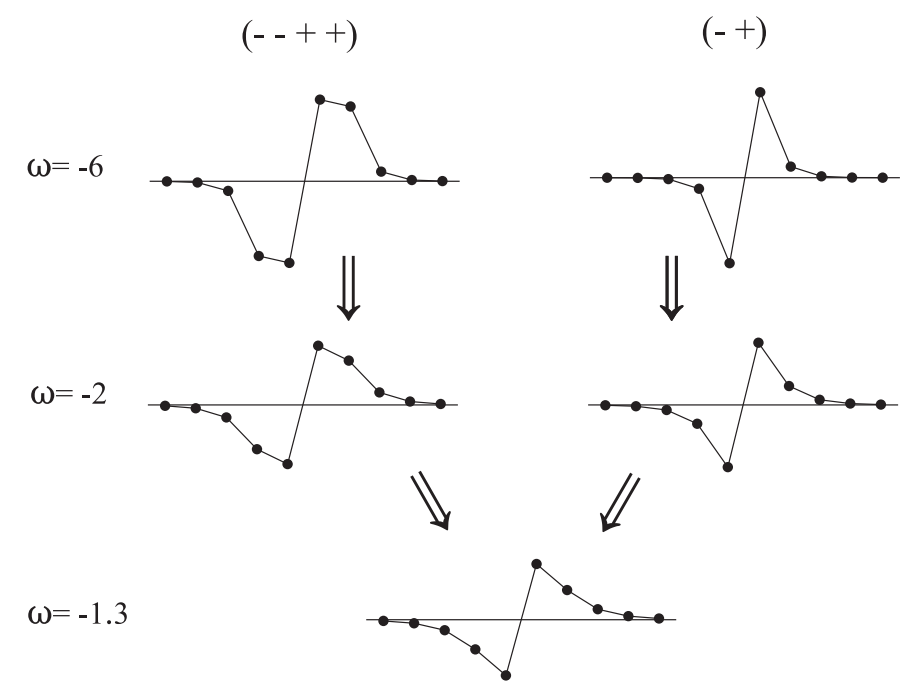

Fig. 12. The saddle-node bifurcation of the solutions with the codes $\{-+\}$ and $\{--++\}$. The point of the bifurcation is $\alpha_{S N} \approx 0.772(\omega \approx-1.295)$.

Let us now consider in more detail the bifurcations of these solutions which occur for $\alpha>\alpha^{*}$. The complete description of the bifurcations for the solutions with codes of length smaller than 4 are shown in Table 1 which was compiled studying directly the transformation of the stable and unstable manifolds $W_{s}$ and $W_{u}$ as $\alpha$ grows. In this Table we show also the bifurcations related to them, for example, pitchfork bifurcations which involves also solutions with codes of length 5 and 6 . It worth noting that the bifurcating solutions may have different lengths of the codes.

In order to study the bifurcations of solutions with greater lengths of the code we used the following approach. First, using the Newton method it is possible to follow numerically the continuation by $\alpha$ of any localized solution of Eq.(5) coded with $\{\xi\}$ from the anticontinuous limit. This can be done until some final point $\alpha=\alpha_{\{\xi\}}$ where the determinant of Newton matrix vanishes. Let at the point $\alpha=\alpha_{\{\xi\}}$ the amplitudes of the sites obtained by continuation of the solution with code $\{\xi\}$ be $v_{n}^{\{\xi\}}\left(\alpha_{\{\xi\}}\right), n \in \mathbb{Z}$. If these final amplitudes are close to each other for two different codes $\left\{\xi_{1}\right\}$ and $\left\{\xi_{2}\right\}$ and at the same time $\alpha_{\left\{\xi_{1}\right\}}$ is close to $\alpha_{\left\{\xi_{2}\right\}}$ then one can expect that the solutions with these codes bifurcate.

We fulfilled this continuation procedure for the solutions of Eq.(5) which have the codes of lengths equal or smaller than 9. Owing to the symmetries we restrict ourselves by the codes which end up by " + ", i.e. $3^{8}=6561$ codes. Using these continuation results we created a database. This database includes the codes $\{\xi\}$ of the solution, the values of $\alpha_{\xi}$ and the values $\left\{v_{n}^{\{\xi\}}\left(\alpha_{\{\xi\}}\right)\right\}$ for large enough number of sites (basically we took 40 sites). The search within this database allows by an introduced code of solution to find a code of another solution which bifurcates with it. The criterion for codes $\left\{\xi_{1}\right\}$ and $\left\{\xi_{2}\right\}$ to be 


\begin{tabular}{|c|c|c|}
\hline$\alpha$ & type & codes \\
\hline 0.324 & $\mathrm{SN}$ & $\begin{array}{ll}\{---+\} \Leftrightarrow\{-0-+\} ; & \{+++-\} \Leftrightarrow\{+0+-\} \\
\{-+++\} \Leftrightarrow\{-+0+\} ; & \{+---\} \Leftrightarrow\{+-0-\}\end{array}$ \\
\hline 0.374 & $\mathrm{SN}$ & $\{+++\} \Leftrightarrow\{+0+\} ; \quad\{---\} \Leftrightarrow\{-0-\}$ \\
\hline 0.485 & $\mathrm{P}$ & $\begin{array}{l}\{+0++\} \Longrightarrow\{++++\} \Longleftarrow\{++0+\} \\
\{-0--\} \Longrightarrow\{----\} \Longleftarrow\{--0-\}\end{array}$ \\
\hline 0.609 & $\mathrm{SN}$ & $\{++++\} \Leftrightarrow\{+00+\} ;\{----\} \Leftrightarrow\{-00-\}$ \\
\hline 0.745 & $\mathrm{P}$ & $\begin{array}{l}\{++-+-\} \Longrightarrow\{+-+-\} \Longleftarrow\{+-+--\} \\
\{--+-+\} \Longrightarrow\{-+-+\} \Longleftarrow\{-+-++\}\end{array}$ \\
\hline $0.745+\Delta$ & $\mathrm{SN}$ & $\{++-+--\} \Leftrightarrow\{+-+-\} ;\{--+-++\} \Leftrightarrow\{-+-+\}$ \\
\hline 0.747 & $\mathrm{P}$ & $\begin{array}{l}\{++-+\} \Longrightarrow\{+-+\} \Longleftarrow\{+-++\} \\
\{--+-\} \Longrightarrow\{-+-\} \Longleftarrow\{-+--\}\end{array}$ \\
\hline $0.747+\Delta$ & $\mathrm{SN}$ & $\{+-+\} \Leftrightarrow\{++-++\} ;\{-+-\} \Leftrightarrow\{--+--\}$ \\
\hline 0.762 & $\mathrm{SN}$ & $\begin{aligned}\{+0-+\} \Leftrightarrow\{+0-++\} ;\{-0+-\} \Leftrightarrow\{-0+--\} \\
\{+-0+\} \Leftrightarrow\{++-0+\} ;\{-+0-\} \Leftrightarrow\{--+0-\}\end{aligned}$ \\
\hline 0.772 & $\mathrm{P}$ & $\begin{array}{l}\{++-\} \Longrightarrow\{+-\} \Longleftarrow\{+--\} \\
\{--+\} \Longrightarrow\{-+\} \Longleftarrow\{-++\}\end{array}$ \\
\hline $0.772+\Delta$ & $\mathrm{SN}$ & $\{-+\} \Leftrightarrow\{--++\} ;\{+-\} \Leftrightarrow\{++--\}$ \\
\hline 0.779 & $\mathrm{P}$ & $\begin{array}{l}\{++--+\} \Longrightarrow\{++--++\} \Longleftarrow\{+--++\} \\
\{--++-\} \Longrightarrow\{--++--\} \Longleftarrow\{-++--\}\end{array}$ \\
\hline $0.779+\Delta$ & $\mathrm{SN}$ & $\{+--+\} \Leftrightarrow\{++--++\} ;\{-++-\} \Leftrightarrow\{--++--\}$ \\
\hline 1.091 & $\mathrm{P}$ & $\begin{array}{l}\{++0-\} \Longrightarrow\{+0-\} \Longleftarrow\{+0--\} \\
\{--0+\} \Longrightarrow\{-0+\} \Longleftarrow\{-0++\}\end{array}$ \\
\hline $1.091+\Delta$ & SN & $\{+0-\} \Leftrightarrow\{++0--\} ;\{-0+\} \Leftrightarrow\{--0++\}$ \\
\hline 1.290 & $\mathrm{P}$ & $\begin{array}{l}\{++00-\} \Longrightarrow\{+00-\} \Longleftarrow\{+00--\} \\
\{--00+\} \Longrightarrow\{-00+\} \Longleftarrow\{-00++\}\end{array}$ \\
\hline $1.290+\Delta$ & $\mathrm{SN}$ & $\{+00-\} \Leftrightarrow\{++00--\} ;\{-00+\} \Leftrightarrow\{--00++\}$ \\
\hline
\end{tabular}

Table 1

Bifurcations of solutions with the code length smaller than 4 and related to them. By the symbol $\Delta$ we denote the difference between the values of $\alpha$ for pitchfork and subsequent saddle-node bifurcations when they are too close to be distinguished by the numerics. 


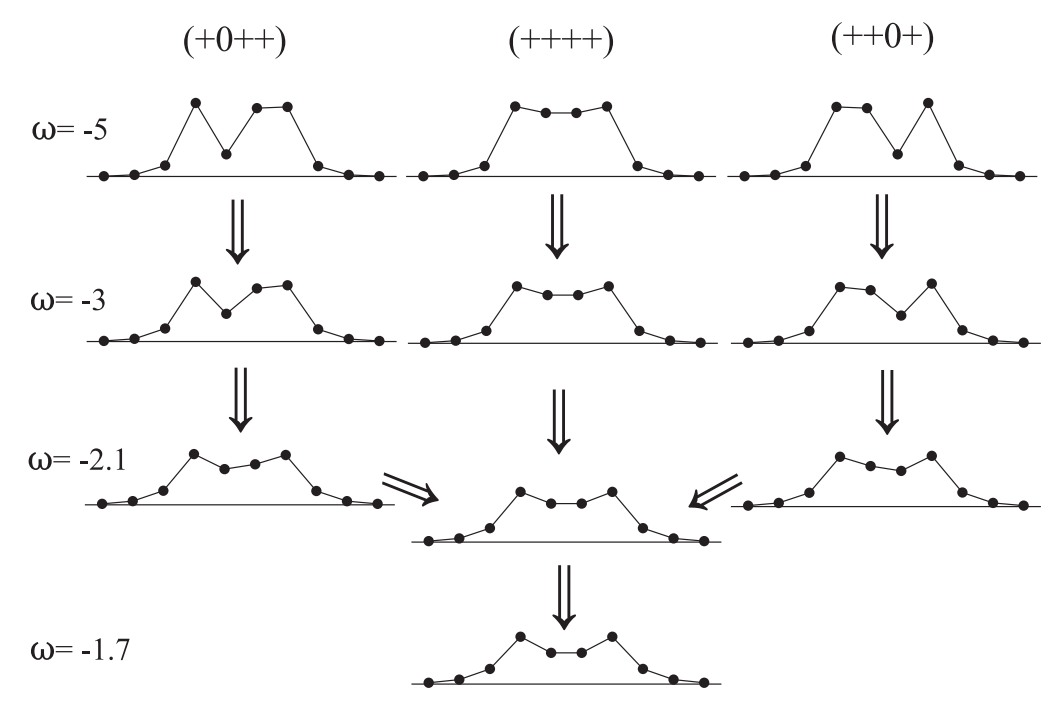

Fig. 13. A pitchfork bifurcation. Two asymmetric solution with the codes $\{+0++\}$ and $\{++0+\}$ merge with the solution with the code $\{++++\}$. The point of the bifurcation is $\alpha_{P} \approx 0.485(\omega \approx-2.062)$.

the codes of bifurcating solutions was taken as follows

$$
\min _{|m|<10}\left(\sum_{n}\left(v_{n-m}^{\left\{\xi_{1}\right\}}\left(\alpha_{\left\{\xi_{1}\right\}}\right)-v_{n}^{\left\{\xi_{2}\right\}}\left(\alpha_{\left\{\xi_{2}\right\}}\right)\right)^{2}<\epsilon\right.
$$

Here $\epsilon$ is some small positive number (we took $\epsilon=0.1$ ).

We test this method using the values of bifurcation points given in Table 1 and in all the cases the discrepancy was about $10^{-3}$ which corresponds to the step in the continuation procedure. In general, the following situations can be distinguished:

- If a solution dies in a saddle-node bifurcation which is not the second bifurcation in the pair "pitchfork - saddle-node" the search in the database gives one code for the solution which bifurcate with it.

- If a solution dies in a pitchfork bifurcation the search in the database typically gives 3 corresponding codes. The reason is that if the initial segment of stable (unstable) manifold is large enough, its lobes become very narrow, so the points of pitchfork bifurcation can be hardly distinguished from the points of subsequent saddle-node bifurcation (in the Table 1 we denote the distance between these close points of bifurcations by the symbol $\Delta$; we should stress that $\Delta$ is not a fixed value but any value which is below the accuracy of the method). So, the search in the database in this case gives two codes of solutions which participate in the pitchfork bifurcation and one more code for the solution which dies in subsequent saddle-node bifurcation.

- By the same reason, if a solution dies in a saddle-node bifurcation which is the second bifurcation in the pair "pitchfork - saddle-node" the search in the database typically gives 3 codes. 


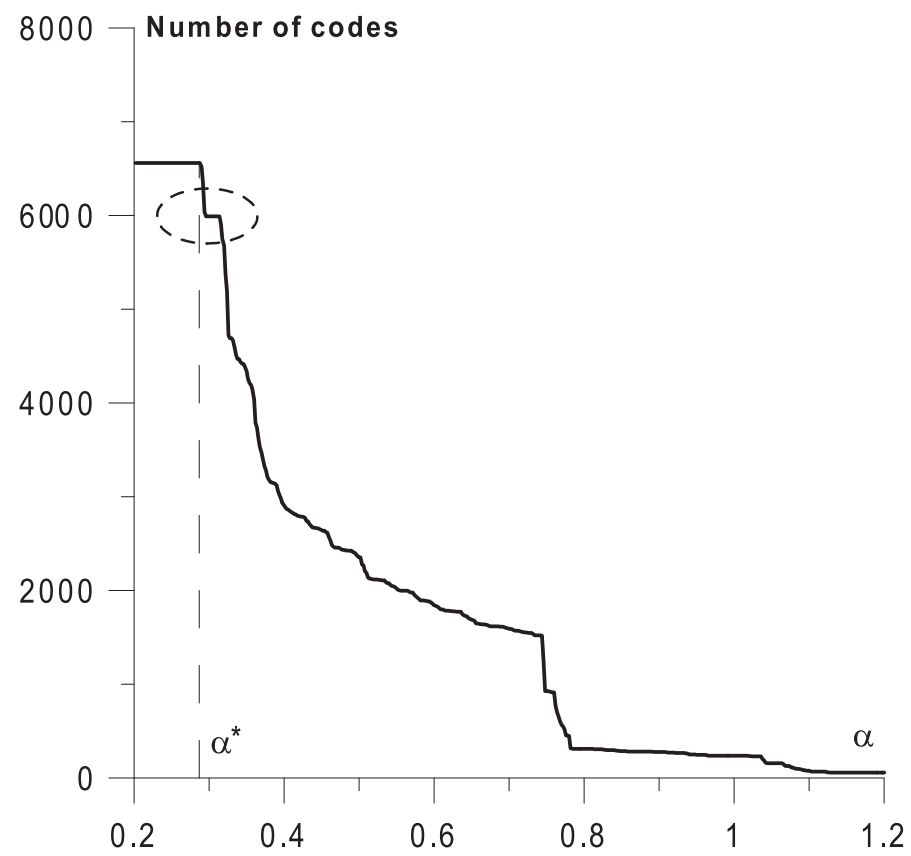

Fig. 14. Number of coexisting solutions with codes smaller or equal to $9, N(\alpha)$, versus $\alpha$.

Also the database allows to calculate the number $N(\alpha)$ of solutions with codes of length smaller or equal to 9 which coexist for a given value of $\alpha$. The plot of $N(\alpha)$ versus $\alpha$ is shown in Fig. 14. The following comments about the bifurcations for $\alpha>\alpha^{*}$ are in order.

(a) There exist intervals of $\alpha$ where no bifurcations occur. One of them is marked in Fig.14 with ellipse. Its boundaries are $\alpha_{1} \approx 0.295$ and $\alpha_{2} \approx 0.314$.

(b) On the interval $\left(\alpha^{*}, \alpha_{1}\right)$ the first sequence of bifurcations occurs. At this interval there die all solutions whose codes contain the sequences of symbols

$$
-+0+-, \quad-+++-, \quad+-0-+, \quad+---+.
$$

This fact is confirmed by the analysis of intersections of stable and unstable manifolds $W_{s}$ and $W_{u}$. The typical bifurcations here are

$$
\begin{aligned}
& \left\{\left\{\eta_{1}\right\}-+++-\left\{\eta_{2}\right\}\right\} \Longleftrightarrow\left\{\left\{\eta_{1}\right\}-+0+-\left\{\eta_{2}\right\}\right\} \\
& \left\{\left\{\eta_{1}\right\}+---+\left\{\eta_{2}\right\}\right\} \Longleftrightarrow\left\{\left\{\eta_{1}\right\}+-0-+\left\{\eta_{2}\right\}\right\} .
\end{aligned}
$$

The end value $\alpha_{1}$ corresponds to the bifurcations

$$
\begin{array}{r}
\left\{\{+-\}^{\infty}-+++-\{-+\}^{\infty}\right\} \Longleftrightarrow\left\{\{+-\}^{\infty}-+0+-\{-+\}^{\infty}\right\} ; \\
\left\{\{-+\}^{\infty}+---+\{+-\}^{\infty}\right\} \Longleftrightarrow\left\{\{-+\}^{\infty}+-0-+\{+-\}^{\infty}\right\}
\end{array}
$$


of the orbits homoclinic to periodic orbit $\{+-\}^{\infty}$. This situation is similar to one found for Hénon map [20]. Following [20] we suppose that for $\alpha_{1}<\alpha<\alpha_{2}$ the localized solutions of Eq.(5) can have any code except ones which includes the words $(20)$.

(c) One more sequence of bifurcations starts with the value $\alpha_{2}$ which corresponds to the bifurcations

$$
\begin{aligned}
& \left\{\{-+\}^{\infty} 0+0\{-+\}^{\infty}\right\} \Longleftrightarrow\left\{\{-+\}^{\infty}++0\{-+\}^{\infty}\right\} ; \\
& \left\{\{+-\}^{\infty} 0+0\{+-\}^{\infty}\right\} \Longleftrightarrow\left\{\{+-\}^{\infty} 0++\{+-\}^{\infty}\right\} ; \\
& \left\{\{+-\}^{\infty} 0-0\{+-\}^{\infty}\right\} \Longleftrightarrow\left\{\{+-\}^{\infty}--0\{+-\}^{\infty}\right\} ; \\
& \left\{\{-+\}^{\infty} 0-0\{-+\}^{\infty}\right\} \Longleftrightarrow\left\{\{-+\}^{\infty} 0--\{-+\}^{\infty}\right\} .
\end{aligned}
$$

These bifurcations affect the solutions which codes contain the sequence of symbols " $-+0+0 "$ and other related to it due to symmetries. At the interval $\left(\alpha^{*}, 0.341\right)$ all these solutions die. The typical bifurcation is

$$
\left\{\left\{\eta_{1}\right\}-+++0\left\{\eta_{2}\right\}\right\} \Longleftrightarrow\left\{\left\{\eta_{1}\right\}-+0+0\left\{\eta_{2}\right\}\right\}
$$

and other related to it by symmetries.

(d) For $\alpha>1.21$ all the solutions in our database have the codes which are composed by the words "+", " - ", "++", " - -" separated by groups of the symbols "0". So, they can be regarded as bound states of Page and SieversTakeno modes. A study of such multipulse solutions for $\alpha$ large can be found in $[21]$.

Since $\omega=-1 / \alpha$ all these results can be rewritten in terms of Eq.(4). In particular one obtains that ACL coding holds for $\omega<\omega^{*} \approx-3.4533$ and the point $\omega^{*}$ is the point of accumulation of saddle-node bifurcations of solutions of Eq.(4).

\section{Conclusion}

To summarize, the main results of the paper are:

(a) For the equation Eq.(4) we used the coding of all localized solutions (3) by the words which consist of the symbols "-", "0" and "+". This coding

comes from anticontinuous limit (ACL-coding). We calculate the boundary of the interval of parameter $\omega, \omega^{*} \approx-3.4533$ for which this coding is valid. 
(b) In order to do this we analysed the problem from the viewpoint of dynamical systems. Since the localized solutions of this discrete equation corresponds to homoclinic points of zero fixed hyperbolic point $\mathcal{O}$ of associated map $T$ we study the mutual position of stable and unstable manifolds of $\mathcal{O}$. A simple rule was found for the ordering of the homoclinic points on these manifolds when anticontinuous approximation is valid.

(c) Based on this we studied the bifurcations of localized solutions of Eq.(4). They correspond to homoclinic bifurcations for the map $T$. It was shown that the end point $\omega^{*}$ of the interval where the ACL-coding is applicable is the point of accumulation of saddle-node homoclinic bifurcations. Then we studied the bifurcations which occur for $\omega>\omega^{*}$. We found an interval of $\omega$ where no bifurcations occur and gave a complete list of bifurcations for the solutions whose coding sequences have lengths smaller or equal to 4.

It worth to compare our results with the results of [12] and [13]. In both these papers Eq.(4) is rewritten in the form

$$
A_{n+1}+A_{n-1}+C A_{n}=3 A_{n}^{3} .
$$

The authors of [12] calculated numerically the values of $C$ for which there exist symmetric localized solutions keeping fixed two internal parameter corresponding to the asymptotic of the solution for $n$ large (specifically, $\epsilon$ and $N)$. The values of these parameters were chosen for reasons of numerical convenience. The coding of solutions introduced in [12], in fact, corresponds to ACL coding. Applying our results one can found that for $C>C^{*} \approx 5.4530$ all solutions of $(21)$ can be coded by $\mathcal{L}_{0}$ sequences. However, all values of $C$ given in resulting Table 1 of [12] exceed this threshold. This explains why the number of found solutions is equal to one predicted by the analysis of number of possible coding sequences (which, generically, does not take place). Changing the internal parameters of the method one can make the value of $C$ smaller than the critical value $C^{*}$ and the situation immediately becomes much more complex. We note also that the illustrative example of [13] related to Eq.(21) corresponds to $C=8$ which also exceeds the threshold value $C^{*}$.

To complete the picture, the information about the existence of discrete breathers should be accompanied with the results on their stability. This part of the work will be presented elsewhere. Here we note that the analysis of bifurcations of discrete breathers proved to be quite useful for the study of their stability. We sketch the relation between these topics as follows. In the linear approximation the stability problem can be reduced to the eigenvalue problem for non-symmetric infinite matrix $L$. This matrix is the product of two infinite symmetric matrices, $L_{-}$and $L_{+}$, where $L_{+}$correspond to linearization of Eq.(4) (Newton matrix) [10]. An information about spectral properties of $L_{-}$and $L_{+}$can be obtained in the anticontinuous limit. Varying $\omega$ one observes 
that at the point of homoclinic bifurcation $\omega=\tilde{\omega}$ (and not in other points) one of the eigenvalues of $L_{+}$vanishes. From the other side one can show that the number of positive eigenvalues of $L_{-}$does not change when $\omega$ varies. These facts, together with the relation between the numbers of positive eigenvalues of $L_{-}, L_{+}$and $L[22,23]$ give a basis for the analysis of stability of discrete breathers.

Authors are grateful to Prof. P.Kevrekidis for reading the manuscript, pointing useful references out and many important comments and to Prof. C. Eilbeck for his illuminating explanations. G.A. thanks the financial support from Spanish Ministry of Education, Culture and Sport through a sabbatical program SAB2000-0340. The work of V.A.B. has been supported by the FCT fellowship SFRH/BPD/5632/2001. V.V.K. acknowledges support from the Programme "Human Potential-Research Training Networks", contract No. HPRN-CT-2000-00158.

\section{A The continuation from anticontinuous limit}

Let us denote

$$
\mathbf{F}(\mathbf{v}, \alpha) \equiv \operatorname{col}\left(\alpha \Delta_{2} v_{n}-v_{n}+v_{n}^{3}\right), \quad n \in \mathbb{Z} .
$$

Then

$$
\frac{d \mathbf{v}}{d \alpha}=-\left[\frac{\partial \mathbf{F}(\mathbf{v}, \alpha)}{\partial \mathbf{v}}\right]^{-1} \frac{\partial \mathbf{F}(\mathbf{v}, \alpha)}{\partial \alpha},
$$

where

$$
\frac{\partial \mathbf{F}(\mathbf{v}, \alpha)}{\partial \alpha}=\operatorname{col}\left(\ldots, \Delta_{2} v_{n-1}, \Delta_{2} v_{n}, \Delta_{2} v_{n+1}, \ldots\right)
$$

and

$$
D_{v} \mathbf{F} \equiv \frac{\partial \mathbf{F}(\mathbf{v}, \alpha)}{\partial \mathbf{v}}=\left(\begin{array}{cccc}
\ddots & \ldots & \ldots & 0 \\
\alpha & \left(-2 \alpha-1+3 v_{n-1}^{2}\right) & \alpha & \ldots \\
\ldots & \alpha & \left(-2 \alpha-1+3 v_{n}^{2}\right) & \alpha \\
0 & \ldots & \ldots & \ddots
\end{array}\right)
$$

are an infinite column-vector and a three-diagonal infinite matrix correspondingly. The continuation by the parameter $\alpha$ is possible while the operator $D_{v} \mathbf{F}$ 
remains invertible. According to [14] this takes place until some value $\alpha=\alpha^{*}$. The paper [14] offers also a method how to estimate the boundary $\alpha^{*}$.

\section{B Proof of Theorem 2.1}

First, we prove the following lemma:

Lemma B.1 For $0<\alpha<\alpha_{0}$, where $\alpha_{0}=(3 \sqrt{3}-1) / 52 \approx 0.0807$ the operator $D_{v} \mathbf{F}$ (see Appendix A) is invertible.

Proof of Lemma B.1. Let us select the conditions when the matrix (A.3) has main diagonal domination (MDD). By definition, MDD means that the modulus of each element on the main diagonal is greater than the sum of module of other elements of the same string i.e.

$$
\delta_{n} \equiv\left|-2 \alpha-1+3 v_{n}^{2}\right|-2 \alpha>0
$$

for any $n \in \mathbb{Z}$. Having MDD situation one can conclude by the generalized Gershgorin theorem that the matrix (A.3) is non-degenerated. Consider the amplitude of one of the sites, $v$, and suppose that $v \geq 0$ (the case $v \leq 0$ can be analyzed by symmetry). The value of $v$ can fall into one of the three intervals:

(i) $0 \leq v \leq 1 / \sqrt{3}$;

(ii) $1 / \sqrt{3} \leq v<1$;

(iii) $v \geq 1$.

The case (i). According to Lemma 2.1

$$
v-v^{3} \leq 4 \alpha \sqrt{1+4 \alpha}
$$

The MDD condition is

$$
\delta \equiv 1-3 v^{2}>0
$$

One can check that the condition (B.2) holds automatically if $0 \leq \alpha<1 / 12$.

The case (ii). Let us denote $\epsilon \equiv 1-v$; then $0 \leq \epsilon<1-1 / \sqrt{3}$. The condition (6) takes the form

$$
\epsilon^{3}-3 \epsilon^{2}+2 \epsilon \leq 4 \alpha \sqrt{1+4 \alpha}
$$


From the other side one has

$$
\delta \equiv\left|-2 \alpha-1+3 v^{2}\right|-2 \alpha \geq 3 v^{2}-1-4 \alpha=3 \epsilon^{2}-6 \epsilon+2-4 \alpha
$$

and the MDD takes place if

$$
3 \epsilon^{2}-6 \epsilon+2-4 \alpha>0 .
$$

One can check that Eq.(B.4) holds automatically if Eq.(B.3) is satisfied and $0 \leq \alpha<\alpha_{0}$; here $\alpha_{0}=(3 \sqrt{3}-1) / 52$ can be found as a solution of the system of equations

$$
\begin{aligned}
\epsilon_{0}^{3}-3 \epsilon_{0}^{2}+2 \epsilon_{0} & =4 \alpha_{0} \sqrt{1+4 \alpha_{0}} ; \\
3 \epsilon_{0}^{2}-6 \epsilon_{0}+2-4 \alpha_{0} & =0 .
\end{aligned}
$$

The simplest way to make sure of the last statement is to plot in the plane $(\epsilon, \alpha)$ the areas corresponding to Eq.(B.3) and Eq.(B.4).

The case (iii). In this case we denote $\epsilon \equiv 1+v$; then $\epsilon>0$. The condition (6) takes the form

$$
\epsilon^{3}+3 \epsilon^{2}+2 \epsilon \leq 4 \alpha \sqrt{1+4 \alpha}
$$

and MDD condition is

$$
\delta \geq 3 v^{2}-1-4 \alpha=3 \epsilon^{2}+6 \epsilon+2-4 \alpha>0 .
$$

One can check that Eq.(B.5) implies Eq.(B.6) for any $\alpha>0$ and $\epsilon>0$. Taking the smallest interval between those found in (i)-(iii) we arrive at the result of Theorem B.1

The proof of Theorem 2.1 follows immediately from Lemma B.1 and Theorem 3.3 of $[1]$.

\section{Some definitions from dynamical systems theory}

Definition C.1 A segment $W_{s}[\mathbf{a}, \mathbf{b}]$ of a stable manifold is the arc of the manifold between points $\mathbf{a}$ and $\mathbf{b}$ which includes these points. Correspondingly, $W_{s}(\mathbf{a}, \mathbf{b})$ is the arc of the manifold between the points $\mathbf{a}$ and $\mathbf{b}$ excluding these points. 
In the same manner one can define the segments $W_{s}[\mathbf{a}, \mathbf{b})$ and $W_{s}(\mathbf{a}, \mathbf{b}]$. The segments on the unstable manifold are defined similarly.

Definition C.2 An initial segment of the branch of a stable manifold, $W_{s}^{ \pm}(\mathcal{O}, \mathbf{a}]$, is the segment of $W_{s}^{ \pm}$which extends from the hyperbolic fixed point $\mathcal{O}$ to the point $\mathbf{a} \in W_{s}^{ \pm}$.

Similarly the initial segment of the unstable manifold, $W_{u}^{ \pm}(\mathcal{O}, \mathbf{a}]$ can be defined.

Definition C.3 A fundamental segment of a branch of a stable manifold, $W_{s}^{ \pm}\left(\mathbf{a}, T^{-1} \mathbf{a}\right], \mathbf{a} \in W_{s}^{ \pm}$is the segment of $W_{s}^{ \pm}$between the point $\mathbf{a}$ and its $T^{-1}$-image, the point $\mathbf{a}$ is excluded and $T^{-1} \mathbf{a}$ is included. Correspondingly, a fundamental segment of the unstable manifold, $W_{u}^{ \pm}(\mathbf{a}, T \mathbf{a}]$, is the segment of $W_{u}^{ \pm}$between the point $\mathbf{a} \in W_{u}^{ \pm}$(excluded) and its T-image (included).

Some comments are in order. The maps $T$ and $T^{-1}$ transform fundamental segments into other fundamental segments. Namely, for any a $\in W_{u}^{ \pm}$(or, correspondingly, $\mathbf{a} \in W_{s}^{ \pm}$)

$$
\begin{aligned}
& T\left\{W_{u}^{ \pm}(\mathbf{a}, T \mathbf{a}]\right\}=W_{u}^{ \pm}\left(T \mathbf{a}, T^{2} \mathbf{a}\right] ; \\
& T\left\{W_{s}^{ \pm}\left(\mathbf{a}, T^{-1} \mathbf{a}\right]\right\}=W_{s}^{ \pm}(T \mathbf{a}, \mathbf{a}] .
\end{aligned}
$$

The whole branch of a stable (unstable) manifold is the union of all its fundamental segments

$$
\begin{array}{ll}
W_{u}^{ \pm}=\bigcup_{n=-\infty}^{\infty} T^{n}\left\{W_{u}^{ \pm}(\mathbf{a}, T \mathbf{a}]\right\}, & \text { for any } \mathbf{a} \in W_{u}^{ \pm} ; \\
W_{s}^{ \pm}=\bigcup_{n=-\infty}^{\infty} T^{n}\left\{W_{s}^{ \pm}(T \mathbf{a}, \mathbf{a}]\right\}, & \text { for any } \mathbf{a} \in W_{s}^{ \pm} .
\end{array}
$$

\section{References}

[1] D. Hennig, G. Tsironis, Phys. Reports 307, 333 (1999).

[2] P. G. Kevrekidis, K. Ø. Rasmussen, A. R. Bishop, Int. J. Mod. Phys. B15, 2833 (2001).

[3] S. Flach, C. R. Willis, Phys. Reports 295, 181 (1998).

[4] J. Ch. Eilbeck, M. Johansson, in "Localization and Energy Transfer in Nonlinear Systems", edited by L. Vázquez, R.S. MacKay, and M.P Zorzano, (World Scientific, Singapore, 2003), p.44; arXiv: nlin.PS/0211049 (2002). 
[5] Abdullaev F. Kh., Baizakov B. B., Darmanyan S. A., Konotop V. V., and Salerno M., Phys. Rev. A 64043606 (2001); G.L.Alfimov, P.G.Kevrekidis, V.V.Konotop and M.Salerno. Phys.Rev.E, 66,(4), 046608 (2002).

[6] G. Kalosakas, S. Aubry, and G. P. Tsironis, Phys. Rev. B 58, 3094 (1998).

[7] A.M. Morgante, M. Johansson, G. Kopidakis et. al., Physica D162, 53 (2002).

[8] A. J. Sievers, S. Takeno, Phys. Rev. Lett. 61, 970 (1988).

[9] J. B. Page, Phys. Rev. B41, 7835 (1990).

[10] E. W. Laedke, O. Kluth, and K. H. Spatschek, Phys. Rev. E54, 4299 (1996).

[11] S. Darmanyan, A. Kobyakov, and F. Lederer, Sov. Phys. JETP 86, 682 (1998).

[12] J. M. Bergamin, T. Bountis, C. Jung, J. Phys.A: Math. Gen. 33, 8059 (2000).

[13] J. M. Bergamin, T. Bountis, M. N. Vrahatis, Nonlinearity 15, 1603 (2002).

[14] R. S. MacKay, S. Aubry, Nonlinearity 7, 1623 (1994).

[15] D. Sterling, H. R. Dullin, and J. D. Meiss, Physica D134, 153 (1999).

[16] H. R. Dullin, J. D. Meiss, Physica D143, 265, (2000).

[17] D. Hennig, N. G. Sun, H. Gabriel, and G. P. Tsironis, Phys. Rev. E 52, 255 (1995);

[18] D. Hennig, K. Ø. Rasmussen, H. Gabriel, and A. Bülow, Phys. Rev. E 54, 5788 (1996).

[19] R. W. Easton, Geometric Methods for Discrete Dynamical Systems, Oxford University Press, 1998.

[20] M. J. Davis, R. S. MacKay, and A. Sannami, Physica D52, 171 (1991).

[21] P. G. Kevrekidis, Phys.Rev. E64, 026611 (2001).

[22] C. K. R. T. Jones, Ergod. Theor. Dynam. Syst. 8, 119 (1988).

[23] M. Grillakis, Comm. Pure. Appl. Math. 41, 747 (1988). 